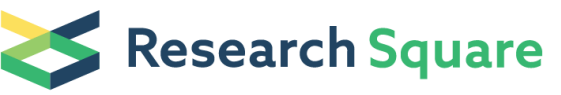

\section{The order of macrophage 4D genome coordinates gene transcription during differentiation and infection}

\section{Gang Cao ( $\sim$ gcao@mail.hzau.edu.cn )}

Huazhong Agricultural University https://orcid.org/0000-0003-0761-7305

\section{Da Lin}

State Key Laboratory of Agricultural Microbiology, Huazhong Agricultural University, Wuhan, China. https://orcid.org/0000-0003-0585-821X

\section{Weize Xu}

Huazhong Agricultural University

\section{Ping Hong}

National Key Laboratory of Crop Genetic Improvement, Huazhong Agricultural University, Wuhan, China.

\section{Chengchao Wu}

State Key Laboratory of Agricultural Microbiology, Huazhong Agricultural University, Wuhan, China.

\section{Zhihui Zhang}

State Key Laboratory of Agricultural Microbiology, Huazhong Agricultural University, Wuhan, China.

\section{Siheng Zhang}

State Key Laboratory of Agricultural Microbiology, Huazhong Agricultural University, Wuhan, China.

\section{Lingyu Xing}

State Key Laboratory of Agricultural Microbiology, Huazhong Agricultural University, Wuhan, China.

\section{Bin Yang}

Huazhong Agricultural University

\section{Wei Zhou}

Huazhong Agricultural University

\section{Qin Xiao}

State Key Laboratory of Agricultural Microbiology, Huazhong Agricultural University, Wuhan, China.

Jinyue Wang

State Key Laboratory of Agricultural Microbiology, Huazhong Agricultural University, Wuhan, China.

\section{Cong Wang}

State Key Laboratory of Agricultural Microbiology, Huazhong Agricultural University, Wuhan, China.

\section{Yu He}

State Key Laboratory of Agricultural Microbiology, Huazhong Agricultural University, Wuhan, China.

\section{Jiangwei Man}

National Key Laboratory of Crop Genetic Improvement 


\section{Xiaofeng Wu}

State Key Laboratory of Agricultural Microbiology, Huazhong Agricultural University, Wuhan, China.

\section{Xingjie Hao}

Huazhong University of Science and Technology

\section{Zhe Hu}

State Key Laboratory of Agricultural Microbiology, Huazhong Agricultural University, Wuhan, China.

\section{Chunli Chen}

Huazhong Agriculture University

\section{Zimeng Cao}

Yangtze University

\section{Rong Yin}

Wuhan University

\section{Zhen Fu}

University of Georgia https://orcid.org/0000-0001-6293-9329

\section{Guoliang Li}

Huazhong Agricultural University https://orcid.org/0000-0003-1601-6640

\section{Article}

Keywords: single-cell Hi-C method, TAD "degree of disorder", genome organization

Posted Date: October 29th, 2021

DOI: https://doi.org/10.21203/rs.3.rs-881693/v1

License: (c) (i) This work is licensed under a Creative Commons Attribution 4.0 International License. Read Full License 

and infection

4 Author names

5 Da Lin ${ }^{1,2,3,4 \# \text {, Weize } \mathrm{Xu}^{1,2,3 \#} \text {, Ping Hong }}{ }^{5,6,7 \#}$, Chengchao $\mathrm{Wu}^{1,2,3}$, Zhihui Zhang ${ }^{1,2,3}$, Siheng Zhang ${ }^{1,2,3}$, 6 Lingyu Xing ${ }^{1,2,3}$, Bing Yang ${ }^{1,2,3}$, Wei Zhou ${ }^{1,2,3}$, Qin Xiao ${ }^{1,2}$, Jinyue Wang ${ }^{1,2}$, Cong Wang ${ }^{1,2,3}$, Yu He ${ }^{1,2,3}$, 7 Jiangwei Man ${ }^{4,5,6}$, Xiaofeng $\mathrm{Wu}^{1,2,3}$, Xingjie $\mathrm{Hao}^{8}$, Zhe $\mathrm{Hu}^{1}$, Chunli Chen ${ }^{9,10}$, Zimeng Cao ${ }^{11}$, Rong Yin ${ }^{12}$, 8 Zhen F. Fu ${ }^{13}$, Guoliang $\mathrm{Li}^{2,4,5,6 *}$ and Gang $\mathrm{Cao}^{1,2,3,4 *}$

\section{Affiliations}

$11{ }^{1}$ State Key Laboratory of Agricultural Microbiology, Huazhong Agricultural University, Wuhan, China.

$12{ }^{2}$ Hubei Hongshan Laboratory, Wuhan, China. ${ }^{3}$ College of Veterinary Medicine, Huazhong Agricultural 13 University, Wuhan, China. ${ }^{4}$ College of Bio-Medicine and Health, Huazhong Agricultural University, 14 Wuhan, China. ${ }^{5}$ National Key Laboratory of Crop Genetic Improvement, Huazhong Agricultural University, 15 Wuhan, China. ${ }^{6}$ Agricultural Bioinformatics Key Laboratory of Hubei Province, Hubei Engineering 16 Technology Research Center of Agricultural Big Data, 3D Genomics Research Center, Huazhong 17 Agricultural University, Wuhan, China. ${ }^{7}$ College of Informatics, Huazhong Agricultural University, Wuhan, 18 China. ${ }^{8}$ School of Public Health, Tongji Medical College, Huazhong University of Science and Technology, 19 Wuhan, China. ${ }^{9}$ College of Life Science and Technology, Huazhong Agricultural University, Wuhan, China.

$20{ }^{10}$ Key Laboratory of Plant Resource Conservation and Germplasm Innovation in Mountainous Region, 21 Guizhou University, Guiyang, China. ${ }^{11}$ College of Animal Sciences, Yangtze River University, Jingzhou, 22 China. ${ }^{12}$ Medical Research Institute, Wuhan University, Wuhan, China. ${ }^{13}$ Department of Pathology, College 23 of Veterinary Medicine, University of Georgia, Athens, GA, USA.

$25 *$ Correspondence to: guoliang.li@mail.hzau.edu.cn; gcao@mail.hzau.edu.cn

$26{ }^{\#}$ These authors contributed equally: Da Lin, Weize Xu, and Ping Hong. 


\section{ABSTRACT}

28 The highly organized three-dimensional genome is crucial for gene transcription. However, it remains elusive how the order of the genome architecture related to its function. Here, we

30 developed a single-cell Hi-C method and proposed TAD "degree of disorder" as a measure of 31 genome organizational patterns, which is correlated with the chromatin epigenetic states, gene expression and co-regulation, and chromatin structure variability in individual cells. Upon Mycobacterium tuberculosis infection, NF- $\mathrm{kB}$ enters into the nucleus, binds to the target genome regions and initiates systematic chromatin conformation reorganization. Furthermore, we identified a remote NF- $\mathrm{kB}$ enriched enhancer promotes the expression of PD-L1 through chromatin loop, which could be a potential anti-tuberculosis and even anti-tumor therapeutic target. The integrated Hi-C, eQTL, and GWAS analysis depicted the atlas of the long-range target genes of tuberculosis susceptible loci. Among which SNP rs1873613 is located in the anchor of a dynamic chromatin loop with LRRK2, whose inhibitor AdoCbl could be an anti-tuberculosis drug candidate. Our study provides comprehensive resources for the 4D genome of immunocytes and sheds insights into the genome organization order and the coordinated gene transcription. 


\section{INTRODUCTION}

It has been many decades since Schrödinger posed the intriguing assumptions about the order or entropy in organisms and the chromosomes containing code-script of the individual's development and function in $1944^{1}$. While the hereditary code-script in the chromosomes has been well deciphered by sequencing now, there is still a big gap to understand the order or entropy of the chromosomes in the cell nucleus. It has been well-established that the folding and organization of the chromosomes in the nucleus is a process with a high degree of non-randomness and order ${ }^{2}$, 3. Paradoxically, recent studies revealed that the three-dimensional (3D) genome architectures fluctuates dynamically among individual cells ${ }^{4}$. This dichotomy provokes the fundamental question how the order and stochasticity of the genome architecture coordinates with the precise transcription of genetic code-script.

In addition to genetic information, the chromosomes are also embedded with dynamic epigenetic codes functioning as blueprints for precise spatial and temporal gene transcription in different developmental stages in response to different stimuli ${ }^{5,6}$. Epigenetic information can be encoded in the sophisticated organization of the 3D genome which can regulate the gene expression in space and time to fulfil distinct physiological tasks ${ }^{7,8}$. Currently, little is known about how the genome organizational patterns fluctuate in different physiological states, especially at single cell level, such as immune cell during differentiation and immunological response. Monocytes are highly plastic immune cells which differentiate to different macrophages and play crucial roles in the first line of defense against pathogens ${ }^{9}$. During differentiation, monocytes transform their nucleus staining pattern ${ }^{10}$, implying a dynamic $3 \mathrm{D}$ chromatin structure. Upon infection, macrophages rapidly mature into different cell subtypes, such as M1 and M2 macrophages, with distinct function and morphology ${ }^{11}$, of which the underlying epigenetic mechanisms are poorly characterized. 
81 leading to approximately 1.3 million deaths and 10.4 million new cases worldwide annually ${ }^{12}$. 82 Upon Mycobacterium tuberculosis (M.tb) invasion into the lung, pulmonary monocytes 83 differentiate into pulmonary macrophages and are activated for immune defense against M.tb 84 infection $^{13}$. Extensive genome-wide association studies (GWASs) have identified many TB 85 susceptibility loci, of which the majority are noncoding regulatory genetic elements ${ }^{14-17}$. However, 86 which genes are the distal regulatory targets of these noncoding loci and how the SNPs influence 87 the 3D genome remains unknown. The aim of this study is, therefore, to use this plastic THP-1 88 monocytes and the differentiated macrophages as a model system to: 1) comprehensively 89 investigate the 4D genome and dynamic epigenetic landscape of macrophages during 90 differentiation and infection at single cell level; 2) investigate how the order and stochasticity of 91 the genome architecture relates to the epigenetic modification and gene transcription. 
Development of single-cell indexed $\mathrm{DLO} \mathrm{Hi}$-C to decipher $3 \mathrm{D}$ genome of THP-1 cells during differentiation and infection

To investigate the 4D genome and epigenetic dynamics during differentiation into different lineages of macrophages, we used THP-1 cells, a highly plastic human monocytic cell line that can be differentiated into macrophage-like cells and activated by M.tb, as the cell model ${ }^{18,19}$. In our study, we named the THP-1 cells before differentiation, after differentiation, and infection by M.tb as Thp1-mono, Thp1-macro, and Thp1-M.tb respectively. After PMA (phorbol 12myristate-13-acetate) treatment, the nuclei of differentiated cells were transformed from ellipsoid to kidney-shaped (Fig. 1a), implying a possible 3D genome conformational change. The distinct morphology alternation of this cell line during different lineages also underlying a rapid reprogramming of gene expression profile during differentiation and infection (Extended Data Fig. 1 a-c and Supplementary Table 1 and 2). Consistent with this observation, the RNA-Seq data indicated that the expression levels of immune-related genes and M1 macrophage marker genes such as CD80, INHBA, CCR7 and TNF-a were significantly upregulated after infection (Extended Data Fig. 1c), indicating that the macrophages were transformed to the M1 phenotype.

During these processes, we observed heterogeneous phenotypes between individual cells, such as some cells have typical macrophages morphology including amoeboid shape, largest surface area and cell volume, while other cells remain as spherical shape during differentiation. Moreover, highly diverse invaded intracellular bacteria numbers and invasion times were observed between different macrophages, suggesting distinct degrees of host immune system activation. To investigate the heterogeneous chromatin architecture of these cells at single cell level, we developed a single-cell-indexed DLO Hi-C (sciDLO Hi-C) based on DLO Hi-C ${ }^{20}$ and two rounds of molecular barcoding to capture the chromatin conformation of individual cells (Fig. 1b). The advantage of this method is that only the $80 \mathrm{bp}$ DNA fragments containing the proximity ligation junction were retained, which can greatly reduce the sequencing noise caused by multiple displacement amplification (Extended Data Fig. 1d-f and Methods). By comparing with other 
121 single-cell Hi-C methods ${ }^{4,21,22}$, we demonstrated that sciDLO Hi-C data-sets contains the highest proportion of proximity ligation junction $\operatorname{reads}^{4}$ (Extended Data Fig. 1e).

123

By sciDLO Hi-C, we obtained 3D genome data of 409 Thp1-mono cells, 424 Thp1-macro cells, and 510 Thp1-M.tb cells (Supplementary Table 3). The territories between different chromosomes can be clearly distinguished based on the simulated 3D genome structure of the individual cells (Extended Data Fig. 1g, h). Next, the single cell data were merged to generate a pooled Hi-C matrix, which is similar to the bulk Hi-C data (Extended Data Fig. 1i). To investigate the heterogeneity of these three type of cells, we employed scHiCTools ${ }^{23}$ to classify all the individual cells based on their 3D genome structures. As shown in Fig. 1c, three distinct clusters of cells were identified, representing Thp1-mono, Thp1-macro, and Thp1-M.tb, respectively. Among which, the Thp1-mono has obvious boundaries between the other two types of cells, whereas Thp1-macro and Thp1-M.tb are partially overlapped. This data reflected that PMA treatment could uniformly reprogram monocyte to a distinct cell type macrophage, whereas macrophage activation caused by M.tb infection was much more heterogeneous. Notably, compared to Thp1-macro and Thp1-mono cells, Thp1-M.tb were enriched with significantly more chromatin contacts around the immune genes (Fig. 1d). For example, the simulated chromatin structures of individual cells showed that the chromatin interaction between innate immune-related genes $N O D 2$ and $B R D 7$ were more contacted in Thp1-M.tb cells (Fig. 1e,f).

\section{TAD degree of disorder (DoD) represents genome organizational patterns and correlates with} gene transcription

To explore the order and stochasticity of genome organization, we further analyzed chromatin contact patterns in individual cells. We observed that while the individual chromatin contacts displayed a certain extent of heterogeneity, the chromatin structures in specific regions of a cell in certain process were uniformly folded. Take the innate immune-related STAT1 gene locus as an example (Fig. 2a-d). Most of the individual Thp1-macro cells had stochastic chromatin interaction 
pattern between STAT1 promoter and an potential enhancer (Fig. 2a,c and Extended Data Fig. 2a), while the promoter-enhancer contact patterns of Thp1-M.tb cells in this region were much more consistent (Fig. 2b,d). These data suggested that in the highly organized genome regions, there was an intrinsic non-randomness or certainty for chromatin folding at both single cell and bulk cell level.

Next, we proposed the concept of TAD degree of disorder (DoD) and developed an algorithm to measure the order and stochasticity of the genome architecture organization of the individual cells (Extended Data Fig. 2b-d and Methods). As only the high order genome loci with similar chromatin interaction in individual cells could exhibit significant interaction spots in the merged Hi-C matrix (Fig. 2e and Supplementary Table 4). We retained all the significant interaction spots in the Hi-C matrix to filter the stochastic genome loci with random chromatin interactions. As the distance between the spots might reflect the similarity of the chromatin folding patterns, the overall mean distance between the spots was then calculated to represent the whole DoD value in the TAD (Extended Data Fig. 2b). As shown in Extended Data Fig. 2e, the closer spot clusters could indeed reflect highly organized chromatin folding patterns. Moreover, the DoD can faithfully indicate the order of the chromatin organization in the individual cells in Fig. 2a-d.

We further calculated the whole genome TAD DoD of the THP-1 cells during differentiation and infection. As illustrated in Extended Data Fig. 2f, the overall TAD DoD distributions in Thp1mono, Thp1-macro, and Thp1-M.tb suggest a dynamic genome architecture alteration during differentiation and infection.

Next, the TAD around MYC gene was used an example to illustrate the detailed DoD dynamics. As this genome locus with abundant enhancer and suppressor elements around the MYC gene is crucial for cell pluripotency and stemness ${ }^{24}$. In Thp1-mono, the chromatin contacts in this region were randomly distributed and had no obvious interaction patterns $(\mathrm{DoD}=2.74)$. Notably, 
the Hi-C matrix of this TAD displayed more highly organized chromatin contact patterns $(\mathrm{DoD}=2.42)$ after differentiation (Fig. 2f), suggesting a systematic non-random promoter-enhancer or promoter-repressor ${ }^{24}$ interaction occurred in the individual cells during differentiation. Notably, we observed a stripe structure right above $M Y C$ gene after differentiation (Fig. 2f, marked with dashed box), which may be attributed to the highly organized chromatin contacts of $M Y C$ gene to a series of cis-elements or genes in this TAD.

Next, we investigated the relationship between the order of the genome architecture organization and the gene expression level in the TAD. As shown in Fig. 2g, the TADs with lower DOD values have generally higher gene expression level in comparison with the more randomly organized TADs with higher DoD in all three cell types. Moreover, the high order TADs with lower DoD values are more enriched in the A compartments, whereas the TADs with higher DoD values are more enriched in the B compartments (Fig. 2h), suggesting the stochasticity of TAD organization is highly related to gene transcription.

As low DoD reflects that the individual cells have similar folding patterns and the chromatin organization is less stochastic, the genes in these TADs may undergo similar transcriptional regulation. In this scenario, the genes in highly organized (low DoD) TADs may be more likely to be synchronously coregulated due to the cooperation within the same transcriptional regulatory complex. To test this hypothesis, we defined the average gene coregulation score (CRS) within a TAD during differentiation and infection, as shown in Extended Data Fig. 2g. Notably, we found that TADs with lower DoDs had overall higher CRSs (Fig. 2i), supporting that highly organized chromatin tends to be more synchronously (or less randomly) coregulated.

Our bulk cell Hi-C data demonstrated that the TADs of the THP-1 cells were underwent with different kind of dynamics during differentiation and infection. Around 30\% of TADs changed their boundaries, including TAD shift, fusion, and division (Extended Data Fig. 2h). For the 
boundaries remained intact TADs, $24.2 \%$ of TAD DoDs decreased, and $3.4 \%$ increased during cell differentiation, whereas $12.0 \%$ decreased and 5.4\% increased during M.tb infection (Extended Data Fig. 2i). As it has been demonstrated that the TAD boundary dynamic could modulate the gene transcription inside TADs ${ }^{25}$, we compared the influence of TAD DoD dynamic with other TAD boundary dynamics on the gene transcription inside the TADs. As shown in Fig. $\mathbf{2 j}$, the dynamic DoD has a more profound influence on gene expression within the TAD than other types of boundary dynamics, including TAD boundary shift, fusion, and division. These results supported the functional significance of TAD DoD.

\section{Active chromatin marks are enriched in the low DOD chromatin regions}

To further investigate the relationship between the organization of genome architecture and chromatin epigenetic modification, we systematically investigated the epigenetic chromatin states across Thp1-mono, Thp1-macro, and Thp1-M.tb cells by ATAC-Seq and comprehensive ChIP-Seq with H3K4me3, H3K4me1, H3K27ac, H3K27me3 and H3K9me3 antibodies. The combination of these analysis revealed the whole genome epigenetic chromatin state and promoter/enhancer state dynamics of THP-1 cells during differentiation and infection (Extended Data Fig. 3a-c). Extended Data Fig. 3d show that the overall gene expression levels are correlated to the promoter activity epigenetic states, supporting the integrity of the epigenetic analysis. Upon M.tb infection, 423 promoters changed from the repressive state to the active state (Extended Data Fig. 3c and Supplementary Table 5). The genes harboring these promoters are enriched in several immune defense pathways (Extended Data Fig. 3e), suggesting that M.tb infection could convert macrophages to the active M1 state by systematical epigenetic reprogramming.

Next, we analyzed the distribution of the active and repressive epigenetic marks in the TADs with different DoDs. Fig. 3a demonstrate that the enhancer and promoter active chromatin marks, such as $\mathrm{H} 3 \mathrm{~K} 4 \mathrm{me} 1, \mathrm{H} 3 \mathrm{~K} 27 \mathrm{ac}$, and $\mathrm{H} 3 \mathrm{~K} 4 \mathrm{me} 3$, are significantly more enriched in the TADs with low DoD values. In contrast, TADs with high DoD contain significantly more transcription 
repressive signals and heterochromatin signals, such as H3K27me3 and H3K9me3 (Fig. 3b). Our data implied that the genome architecture of TADs with low DoDs were more sophisticatedly organized, which might be mediated by the elegant cooperation with a greater number of transcriptional regulatory factors and more accessible chromatin within TADs. Consistent with this hypothesis, we observed that the DoD was indeed highly negatively correlated with ATAC signals within the TADs (Fig. 3c), suggesting that the DoD is correlated to the chromatin accessibility and the subsequent binding of transcriptional regulatory proteins.

\section{HERC5 and HERC6 are IFN-induced HECT-type E3 protein ligases involved in anti-bacterial} defense $^{26}$. We observed that the TAD DoD value of HERC5 and HERC6 gene regions were significantly decreased upon M.tb infection, suggesting a refining of the genome architecture organization (Fig. 3d,e). Moreover, the ATAC peak and active chromatin marks such as H3K4me3 and H3K27ac were enriched in the promoter region. Consistently, the expression of HERC5 and HERC6 were also synchronously co-upregulated (Fig. 3f,g). By simulating the spatial structure of this region, we found that in the low DOD state, the spatial distance between HERC5 and HERC6 was more adjacent (Fig. 3h,i), suggesting these two genes have the tendency to cooperate with each other and be co-regulated by the same transcription regulatory complex in response to the infection.

Our bulk and single cell DLO Hi-C data suggested that the TADs with low DoD display a high degree of certainty and consistence of the local chromatin folding patterns among individual cells. Thus, the TAD "degree of disorder" may reflect the entropy of the chromatin organization in a TAD. The highly organized TADs with lower "TAD entropy" levels have overall lower DoD values, more active enhancer/promoter enrichment, higher gene transcription levels and more concerted coregulation. In contrast, the disordered TADs with higher "TAD entropy" levels generally exhibit lower transcription and more random coregulation (Fig. 3j). Together, these results suggested that the order of chromatin structure is highly correlated with its epigenetic 
modification and gene transcription.

Remodeling of the chromatin configuration around GBP family genes orchestrates their coexpression upon M.tb infection

To further investigate local TAD DoD in details, we explored the genome sites with dynamic DoD, such as the TAD with the guanylate binding protein (GBP) gene family, of which the DoD was significantly decreased upon M.tb infection in the bulk cell DLO Hi-C data (Fig. 4a). The single-cell chromatin interaction matrix with high quality in this $G B P$ region were merged as shown in Fig. 4b, in which each color represented the chromatin contact from the same individual cells. We calculated the DoD based on the merged chromatin interaction matrix from sciDLO HiC data and found that the DoD was decreased after M.tb infection, which is consistent with the results from bulk cell $\mathrm{Hi}-\mathrm{C}$ data.

By virtue of the merged single cell contact matrix, we found that the chromatin contacts fluctuate considerably among individual cells, suggesting heterogenous genome organizational patterns at single cell level. However, upon infection this TAD has smaller DoD and more intrinsically organized local chromatin interaction patterns in comparison to the random pattern in the Thp1-macro cells with high DoD (Fig. 4b). For example, a series of genome loci in this TAD were sequentially interacted with GBP5 respectively among individual Thp1-M.tb cells, whereas the chromatin contact pattern of this location in Thp1-macro cells was much more random (Fig. 4c). As one Hi-C experiment can only capture chromatin interaction at single time-point, the merged interactions of GBP5 with other loci from the individual cells may reflect the chromatin interactions around GBP5 from different time-points. As they all looped with GBP5, it likely that these genes are all assembled in a transcription factory.

Consistent with our observation that DoD is negatively correlated with gene co-regulation, Fig. 4a demonstrated that the expression levels of the GBP1-5 in the TAD was synchronously co- 
upregulated. The immunostaining assay showed that GBP family proteins assembled tightly around the surface of M.tb cells probably to prevent its spreading (Extended Data Fig. 4a). These observations may suggest that, in response to M.tb infection, this relatively chaotic TAD with high DoD or entropy became highly organized. This may be coordinated by the opening of specific genome loci and the subsequent binding of corresponding proteins which formed new chromatin loops that linked the genes into an active transcription factory. In this way, it could efficiently achieve synchronous co-transcription of these defense-related genes.

Transcription factory tends to form liquid-liquid phase separation (LLPS) condensates ${ }^{27}$ to efficiently activate gene transcription. It has been shown that super enhancer (SE) binding protein MED1 and BRD4 undergo phase separation and can be used as phase separation marker proteins within transcription factory ${ }^{28}$. We then test whether GBP family gene region is relocated into LLPS zone in the process of immune activation. Based on previous BRD4 and MED1 ChIP-seq data ${ }^{29}$, we found that GBP family genome region were enriched with these two phase separation marker proteins (Fig. 4d). Our ATAC-seq and H3K4me3 ChIP-seq data indicate that the chromatin state is activated and may bind with more regulatory proteins after infection (Fig. 4a). Furthermore, the integrated Hi-C chromatin loop and BRD4 and MED1 ChIP-seq analysis suggest that the BRD4 and MED1 occupied SE are spatially in close proximity to all the GBP family gene loci after M.tb infection (Fig. 4d).

To further confirm phase separation in GBP gene family region, we performed co-staining of BRD4 and MED1 (by Immunofluorescence) with GBP gene family region (by fluorescence in situ hybridization). As shown in Fig. 4e and Extended Data Fig. 4b, the overlapping ratio of BRD4 and MED1 puncta and the DNA-FISH signal of GBP gene family region is significantly increased after infection, suggesting the GBPs region tends to relocate into LLPS transcription factory zone to efficiently initiate these immune-defense gene expression. Together, our data implied that macrophages could dynamically adapt their 3D genome structure and coordinate with LLPS 
transcription factory to efficiently express these immune-defense gene to fulfil distinct physiological functions during immunogical response.

\section{NF-kB initiates systematic chromatin remodeling of its target genome regions during M.tb} infection

During differentiation and M.tb infection, the overall DoD were gradually decreased, which is in line with the plasticity of these cells (Fig. 5a and Extended Data Fig. 5a). In these processes, the reduction of DoD was accompanied with the increase of chromatin loops, suggesting that more loop mediated cis-element interaction occurs in low DoD region (Fig. 5b). Upon infection, about 1,864 loops were strengthened. These chromatin loops related genes are listed in Supplementary Table 6. Notably, these genes are significantly enriched in immunity pathways, which is not observed in the genes located in the weakened loops (Extended Data Fig. 5b). The dynamic immunity related enhancer-gene regulatory network in Chr12 during infection was shown in Extended Data Fig. 5c. The KEGG analysis of strengthened loop anchor genes (Supplementary Table 6) showed that, 5 of the top 10 enriched pathways were directly related to NF- $\mathrm{BB}$ signaling pathways (Extended Data Fig. 5d). Furthermore, a large number of NF- $\kappa B$ binding motifs were enriched around the transcription start site (TSS) of these strengthened loop anchor genes (Fig. 5c), suggesting that NF- $\kappa \mathrm{B}$ participates in chromatin remodeling during M.tb infection. The immunofluorescence assay revealed that NF- $\kappa \mathrm{B}(\mathrm{p} 65)$ was indeed translocated into the nucleus upon infection (Fig. 5d).

The chromatin accessibility and RNA expression analysis showed that, upon M.tb infection, the NF- $\mathrm{NB}$ target loci turned to be more open and the expression level of target genes was significantly up-regulated compared to the random genes (Fig. 5e). Moreover, the chromatin loops related to the NF- $\kappa \mathrm{B}$ target genes were more strengthened compared to the random genes (Fig. 5f). We further investigated the chromatin remodeling of the typical NF- $\kappa \mathrm{B}$ target gene loci, such as IFITs, CCLs, GBPs, HERCs, NFKB1, and TNFSF10. Fig. 5g demonstrated that upon infection, a 
greater number of loops were formed in these regions and the corresponding DoD was also reduced. This data was further validated by ChIP-PCR, showing that the NF- $\kappa$ B was significantly enriched in the loop anchor regions of IFIT3, CCL2, GBP4, and HERC2 upon infection (Extended Data Fig. 5e). These evidences suggest that, upon infection, NF- $\kappa B$ translocated into nucleus, bound to specific target regions, readjusted the local DoD by reorganizing the chromatin structure for a concerted transcription of the defense genes.

IFIT gene loci are NF- $\mathrm{KB}$ target gene sites ${ }^{30}$, of which the DoD was decreased upon infection. The single cell Hi-C data also demonstrated that the local chromatin interaction pattern became more ordered during infection (Fig. 5h). The chromatin loop analysis revealed that these IFIT genes were regulated by a single upstream cis element enriched with NF- $\kappa$ B binding motif (Fig. $\mathbf{5 i , j}$ ). Before infection, the cis-element was linked to IFIT1B and IFIT5 (Fig. 5i), which were dynamically reshuffled to link with IFIT1, IFIT2, and IFIT3 after infection (Fig. 5j). Interestingly, it has been demonstrated that IFIT1, IFIT2, and IFIT3 proteins can interact with each other and form a complex to perform antipathogenic functions ${ }^{31}$. Consistently, we observed synchronously upregulated expression of these three genes in the newly established loops (Fig. 5j). Notably, IFIT1B and IFIT5, which are not located in this spatially adjacent hub, were not upregulated. These data demonstrated a high order chromatin structure mediated elegant spatial and temporal coregulation of the NF- $\kappa \mathrm{B}$ target genes transcription during immunoresponse (Fig. 5k).

\section{A remote NF-KB enriched enhancer promotes the expression of PD-L1 through chromatin loop}

After engulfing M.tb, macrophages can present $M . t b$ antigens via major histocompatibility complex (MHC) molecules to T-cells to eliminate M.tb infection ${ }^{32}$. During this process, M.tb also evolves various strategies to escape immunological clearance. In this scenario, we observed immune checkpoint protein PD-L1, which can counteracting $\mathrm{T}$ cell-activating signals, was dramatically upregulated after M.tb infection (Extended Data Fig. 1c). Through the Hi-C contact matrix, we identified a putative enhancer region highly enriched with $\mathrm{H} 3 \mathrm{~K} 4 \mathrm{me} 3$ modification 
(Chr9: 4,760,070-4,769,779) contact with the PD-L1 promoter through a Hi-C loop (Fig. 6a-c). Notably, the ATAC-seq data showed that these enhancer and promoter regions were more accessible and enrichment active chromatin modifications after M.tb infection (Fig. 6b,c), indicates that more regulatory proteins are enriched in the enhancer region. Transcription factor binding motif analysis revealed that both enhancer and promoter (Fig. 6b,c and Extended Data

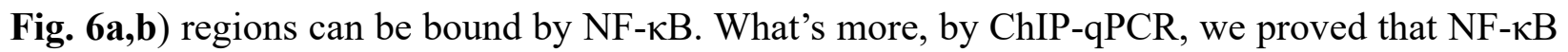
(p65) was significantly enriched in this enhancer region after infection (Fig. 6d).

Since NF- $\kappa \mathrm{B}$ can directly induces $P D-L 1$ gene transcription by binding to its promoter ${ }^{33}$, we speculate that this enhancer region can directly regulate the $P D-L 1$ gene expression mediated by chromatin loop. To further confirm the function of PD-L1 enhancer, we knocked out this enhancer region in the THP-1 cell line by the CRISPR/Cas9 system and validated its regulatory function (Fig 6e and Extended Data Fig. 6c-f). As predicted, the qPCR and western blot data demonstrated that knockout of the $P D-L 1$ enhancer can indeed attenuate the upregulation of PD-L1 expression at both mRNA and protein levels upon M.tb infection (Fig. 6f,g). Collectively, these results suggested a synchronous opening of the enhancer and promoter regions for transcription factor binding and activated $P D-L 1$ gene transcription during M.tb infection (Fig. 6h). This PD-L1 enhancer has potential to become anti-tuberculosis and even anti-tumor therapeutic target. It would be of great interest to further investigate how M.tb infection reprogram the epigenetic code in this enhancer locus and what is physiological role of this modification in the pathogenesis of M.tb.

Integrated omics analysis of long-range target genes of TB susceptibility loci via chromatin looping

To obtain a comprehensive map of tuberculosis (TB) susceptibility loci and their longrange regulatory gene targets via chromatin looping, we collected all reported TB susceptibility loci ${ }^{14-17}$ and performed integrated omics analysis of GWAS, eQTL, and Hi-C (Fig. 7a, Extended Data Fig. 7a, and Supplementary Table 7). The comprehensive TB susceptibility loci and their 
target genes through long-range chromatin interactions were shown in Extended Data Fig. 7b. Of note, the chromatin interaction loops between the LRRK2, NSL1, and ASAP1 and the corresponding TB susceptibility loci were significantly strengthened during M.tb infection (Fig. 7b and Extended Data Fig. 7c,d). Importantly, the target genes of TB susceptibility loci discovered by this integrated omics analysis, such as $A S A P 1$ and $L R R K 2$, have been reported to be involved in TB pathogenesis ${ }^{17,34}$, supporting the integrity of our multiomics analysis.

We further analyzed $L R R K 2$, which has a strong loop and eQTL correlation with the TB susceptibility SNP rs1873613. The DoD in the TAD was decreased after M.tb infection (Fig. 7b). Moreover, the simulated chromatin structure revealed that SNP rs1873613 and LRRK2 tended to be adjacent in space upon infection (Fig. 7c). The ENCODE ChIP-Seq data showed that this SNP is located in the enhancer region of $L R R K 2$ with the binding site of STAT3, SPI1, and FOS (Extended Data Fig. 7e). Moreover, this SNP is located right in the anchor of an $L R R K 2$ chromatin loop that was significantly strengthened after infection (Fig. 7b). This data suggests that this TB susceptibility SNP locus was dynamically reshuffled upon infection to spatially link with $L R R K 2$ to regulate its gene transcription. Consistently, the eQTL analysis data showed that a T:C mutation can indeed increase the expression of $L R R K 2$ in the lungs (Fig. 7d). This SNP in the patient may possibly influence the contact of $L R R K 2$ and the enhancer and therefore disturb the transcription regulation of $L R R K 2$.

It has been demonstrated that LRRK2 promoted the proliferation of M.tb by inhibiting phagosomes maturation $^{34}$, suggesting LRRK2 might be a TB drug target. Thus, we investigated the anti-M.tb effect of AdoCbl, an inhibitor of LRRK2 ${ }^{35}$. As shown in Fig. 7e-g, AdoCbl can indeed promote the maturation of phagosomes in TB-infected bone marrow-derived macrophages and decrease the colony-forming unit count of intracellular M.tb. Furthermore, the in vivo experiment showed that AdoCbl could significantly inhibit the proliferation of M.tb in the lungs (Fig. 7h,i). This result was further supported by hematoxylin-eosin staining of tissue sections, showing that 
AdoCbl significantly inhibited the initiation of lung and spleen lesions (Fig. 7j). These data revealed that the TB susceptibility SNP (rs1873613) in the dynamic anchor of LRRK2 enhancer loop could promote the expression of LRRK2 through chromatin looping. It subsequently represses the clearance of M.tb by inhibiting phagosome maturation and thus causes susceptibility to $\mathrm{TB}$, of which this process can be attenuated by a potential TB drug candidate, AdoCbl (Extended Data Fig. 7f).

Collectively, we delineated the 4D genome landscape of THP-1 cells during differentiation and infection at single cell resolution. Our data showed that the immunological enhancer-promoter loops, especially the NF-kB target regions, were reorganized to orchestrate synchronous defense gene transcription for M.tb clearance. It provides a comprehensive resource for epigenetic regulation of immunocytes and for M.tb infection studies, such as anti-M.tb drug screening and the TB pathogenesis mechanisms of the patients with susceptibility SNPs. Importantly, we proposed TAD DoD to measure the genome organizational patterns, which is correlated with the chromatin epigenetic states, chromatin structure variability in individual cells, expression and coregulation of the genes within the $\mathrm{TAD}$, supporting the order and stochasticity of genome architecture is related to its function. These data shed insights into the dynamic genome organizational patterns at single cell level and illustrated how the 4D genome coordinates gene transcriptional programs during differentiation or in response to different stimulus. 


\section{References}

439 1. Schrodinger, E. What is life? The physical aspect of the living cell. (At the University Press, 1951).

$4412 . \quad$ Misteli, T. Beyond the sequence: cellular organization of genome function. Cell 128, 787800 (2007).

443 3. Rowley, M.J. \& Corces, V.G. Organizational principles of 3D genome architecture. Nature Reviews Genetics 19, 789-800 (2018).

445 4. Ulianov, S.V. et al. Order and stochasticity in the folding of individual Drosophila genomes. Nature Communications 12, 1-17 (2021).

447 5. Kanherkar, R.R., Bhatia-Dey, N. \& Csoka, A.B. Epigenetics across the human lifespan. Front. Cell Dev. Biol. 2, 49 (2014).

449 6. Meissner, A. Epigenetic modifications in pluripotent and differentiated cells. Nat. Biotechnol. 28, 1079-1088 (2010).

$4517 . \quad$ Misteli, T. The Self-Organizing Genome: Principles of Genome Architecture and Function. Cell (2020).

453 8. Mourad, R. \& Cuvier, O. Predicting the spatial organization of chromosomes using epigenetic data. Genome Biol. 16, 182 (2015).

455 9. Garg, S.K., Vitvitsky, V., Gendelman, H.E. \& Banerjee, R. Monocyte differentiation, 456 activation, and mycobacterial killing are linked to transsulfuration-dependent redox metabolism. J. Biol. Chem. 281, 38712-38720 (2006). 
458 10. Skinner, B.M. \& Johnson, E.E. Nuclear morphologies: their diversity and functional 459 relevance. Chromosoma 126, 195-212(2017).

460 11. Ivashkiv, L.B. Epigenetic regulation of macrophage polarization and function. Trends Immunol. 34, 216-223 (2013).

462 12. World Health Organization Global Tuberculosis Report 2018. (World Health Organization, $463 \quad$ Geneva; 2018).

464 13. Shi, C. \& Pamer, E.G. Monocyte recruitment during infection and inflammation. Nat. Rev. Immunol. 11, 762-774 (2011).

466 14. Thye, T. et al. Genome-wide association analyses identifies a susceptibility locus for 467 tuberculosis on chromosome 18q11.2. Nat. Genet. 42, 739-741 (2010).

468 15. Thye, T. et al. Common variants at $11 \mathrm{p} 13$ are associated with susceptibility to tuberculosis. Nat. Genet. 44, 257-259 (2012).

470 16. Zheng, R. et al. Genome-wide association study identifies two risk loci for tuberculosis in Han Chinese. Nat. Commun. 9, 4072 (2018).

472 17. Curtis, J. et al. Susceptibility to tuberculosis is associated with variants in the ASAP1 gene encoding a regulator of dendritic cell migration. Nat. Genet. 47, 523-527 (2015).

474 18. Phanstiel, D.H. et al. Static and dynamic DNA loops form AP-1-bound activation hubs 475 during macrophage development. Mol. Cell 67, 1037-1048.e1036 (2017).

476 19. Auwerx, J. The human leukemia cell line, THP-1: a multifacetted model for the study of 477 monocyte-macrophage differentiation. Experientia 47, 22-31 (1991). 
478 20. Lin, D. et al. Digestion-ligation-only Hi-C is an efficient and cost-effective method for 479 chromosome conformation capture. Nat. Genet. 50, 754-763 (2018).

480 21. Flyamer, I.M. et al. Single-nucleus Hi-C reveals unique chromatin reorganization at 481 oocyte-to-zygote transition. Nature 544, 110-114 (2017).

482 22. Tan, L., Xing, D., Chang, C.-H., Li, H. \& Xie, X.S. Three-dimensional genome structures 483 of single diploid human cells. Science 361, 924-928 (2018).

484 23. Li, X., Feng, F., Leung, W.Y. \& Liu, J. scHiCTools: a computational toolbox for analyzing 485 single-cell Hi-C data. bioRxiv, 769513 (2020).

24. Fulco, C.P. et al. Systematic mapping of functional enhancer-promoter connections with CRISPR interference. Science 354, 769-773 (2016).

25. Arzate-Mejía, R.G., Cerecedo-Castillo, A.J., Guerrero, G., Furlan-Magaril, M. \& RecillasTarga, F. In situ dissection of domain boundaries affect genome topology and gene transcription in Drosophila. Nature communications 11, 1-16 (2020).

26. Wong, J.J.Y., Pung, Y.F., Sze, N.S.-K. \& Chin, K.-C. HERC5 is an IFN-induced HECTtype E3 protein ligase that mediates type I IFN-induced ISGylation of protein targets. Proceedings of the National Academy of Sciences 103, 10735-10740 (2006).

27. Palikyras, S. \& Papantonis, A. Modes of phase separation affecting chromatin regulation. Open biology 9, 190167 (2019).

28. Sabari, B.R. et al. Coactivator condensation at super-enhancers links phase separation and gene control. Science 361 (2018). 
498 29. Angus, S.P. et al. FOXA1 and adaptive response determinants to HER2 targeted therapy in 499 TBCRC 036. NPJ breast cancer 7, 1-15 (2021).

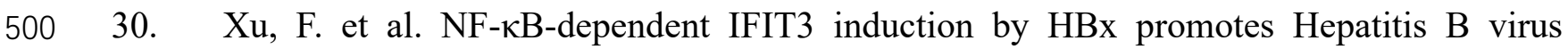
501 replication. Frontiers in microbiology 10, 2382 (2019).

502 31. Fleith, R.C. et al. IFIT3 and IFIT2/3 promote IFIT1-mediated translation inhibition by 503 enhancing binding to non-self RNA. Nucleic Acids Res. 46, 5269-5285 (2018).

504 32. Harding, C.V. \& Boom, W.H. Regulation of antigen presentation by mycobacterium 505 tuberculosis: a role for toll-like receptors. Nat. Rev. Microbiol. 8, 296-307 (2010).

506 33. Antonangeli, F. et al. Regulation of PD-L1 Expression by NF- $\kappa \mathrm{B}$ in Cancer. Frontiers in 507 immunology 11 (2020).

508 34. Härtlova, A. et al. LRRK2 is a negative regulator of Mycobacterium tuberculosis 509 phagosome maturation in macrophages. EMBO J. 37, e98694 (2018).

35. Schaffner, A. et al. Vitamin B12 modulates Parkinson's disease LRRK2 kinase activity through allosteric regulation and confers neuroprotection. Cell Res. 29, 313-329 (2019). 


\section{Materials and Methods}

\section{Cell culture and mycobacterial infection}

516 THP-1 cells (ATCC, TIB-202) were grown in RPMI-1640 containing $10 \%$ FBS. To differentiate

517 the monocytes to macrophages, cells were treated with PMA (phorbol 12-myristate 13-acetate)

518 (final concentration $=40 \mathrm{ng} / \mu \mathrm{l}$ ) for $48 \mathrm{~h}$ at $37^{\circ} \mathrm{C}$ in a $5 \% \mathrm{CO}_{2}$ atmosphere, and then washed with

519 pre-warmed PBS and incubated with fresh culture medium for another 24 h. For M.tb infection 520 experiment, H37Ra was pelleted (4,000 rpm, RT, $10 \mathrm{~min}$ ), washed twice with RPMI-1640, 521 resuspended in $1 \mathrm{ml}$ THP-1 culture medium, and dispersed using BD insulin syringes (BD, 522 328421). Monocyte derived macrophages (Thp1-macro) were infected with H37Ra at MOI 523 (multiplicity of infection) 20. Four hours later the infected cells were washed twice with pre524 warmed PBS and incubated with fresh culture medium for another $8 \mathrm{~h}$.

\section{RNA-Seq library preparation}

527 RNA was extracted using the RNAiso Plus (Takara, 9109) according to manufacturer's protocol. 528 Sequencing libraries were prepared using the VAHTS Stranded mRNA-Seq Library Prep Kit 529 (Vazyme, NR602-02) according to manufacturer's protocol.

\section{ChIP-Seq library preparation}

$5324 \times 10^{6}$ cells and $3 \mu \mathrm{g}$ antibody were used per immunoprecipitation. Antibody of H3K4me1 (Abcam, 533 ab8895), H3K4me3 (Abcam, ab8580), H3K9me3 (Abcam, ab8898), H3K27ac (Abcam, ab4729), 534 and H3K27me3 (Millipore, 07-449) were used in this study. The ChIP DNA was obtained using 535 SimpleChIP ${ }^{\circledR}$ Enzymatic Chromatin IP Kit (CST, \#9003). After immunoprecipitation, ChIP-seq 536 library was constructed using NEBNext® Ultra ${ }^{\mathrm{TM}}$ DNA Library Prep Kit (NEB, E7370S).

\section{ATAC-Seq library preparation}

$5391 \times 10^{5}$ cells were centrifuged at $800 \mathrm{~g}$ for $5 \mathrm{~min}$ and then washed once using $500 \mu \mathrm{l}$ of cold $1 \times$ PBS and centrifuged at $800 \mathrm{~g}$ for another $5 \mathrm{~min}$. Cells were lysed using cold lysis buffer (10 mM Tris- 
$\mathrm{HCl}\left(\mathrm{pH} 8.0\right.$ at $\left.25^{\circ} \mathrm{C}\right), 10 \mathrm{mM} \mathrm{NaCl}, 0.3 \%$ Igepal CA-630). After lysis, nuclei were spun down at $800 \mathrm{~g}$ in $4{ }^{\circ} \mathrm{C}$ for $10 \mathrm{~min}$. The pellet was resuspended in the transposase reaction mix $(10 \mu 15 \times$ TTBL buffer (Vazyme, TD501-02), $5 \mu$ TTE Mix V50 buffer (Vazyme, TD501-02) and $35 \mu 1$ $\mathrm{ddH}_{2} \mathrm{O}$ water). Tagmentation was carried out for $10 \mathrm{~min}$ at $55{ }^{\circ} \mathrm{C}$. Immediately following transposition, the DNA was purified using a Qiagen MinElute PCR Purification Kit (Qiagen, 28004) and eluted with $10 \mu$ elution buffer. The ATAC-Seq library was amplified by the primers in TruePrepTM Index Kit V2 for Illumina ${ }^{\circledR}$ (Vazyme, TD202).

\section{In situ DLO Hi-C experiment}

In situ DLO Hi-C was performed as previously described ${ }^{20}$. Five million cells were double crosslinked with 1.5 mM EGS (Thermo) and 1\% formaldehyde (Sigma) and lysed in lysis buffer (10 $\mathrm{mM}$ Tris- $\mathrm{HCl}\left(\mathrm{pH} 8.0\right.$ at $\left.25^{\circ} \mathrm{C}\right), 10 \mathrm{mM} \mathrm{NaCl}, 0.3 \%$ Igepal CA-630, 0.5\% SDS, and complete protease inhibitor (Roche)), incubated at $60^{\circ} \mathrm{C}$ for $5 \mathrm{~min}$, and placed on ice immediately. After incubation, the nuclei were pelleted by centrifugation at 1,000 r.p.m. for $5 \mathrm{~min}$ and washed them once with ice-cold PBS. A total of $310 \mu \mathrm{l}$ of ddH2O, $20 \mu \mathrm{l}$ of $20 \%$ Triton $\mathrm{X}-100,40 \mu \mathrm{l}$ of $10 \times$ NEBuffer 2.1, and $30 \mu \mathrm{l}$ of MseI (NEB, 10 units/ $\mu$ l) was then added to the nuclei and incubated for $6 \mathrm{~h}$ at $37{ }^{\circ} \mathrm{C}$ with rotation at 15 r.p.m. After restriction enzyme digestion, $50 \mu \mathrm{l}$ of MseI half linkers $(600 \mathrm{ng} / \mu \mathrm{l}), 5 \mu \mathrm{l}$ of $100 \mathrm{mM}$ ATP, $20 \mu \mathrm{l}$ of T4 DNA ligase (Thermo, 5 units/ $\mu \mathrm{l})$, and $25 \mu \mathrm{l}$ of ddH2O were added to the $400 \mu \mathrm{l}$ of digested chromatin and mixed thoroughly. The mixture was then incubated at $25^{\circ} \mathrm{C}$ for $1 \mathrm{~h}$ with rotation at 15 r.p.m. After half-linker ligation, the nuclei were centrifuged at $4{ }^{\circ} \mathrm{C}$ for 5 min at 1000 r.p.m. and washed twice with $1 \mathrm{ml}$ of ice-cold PBS. The linker-ligated nuclei were gently resuspended in $200 \mu \mathrm{l}$ of $1 \times$ T4 DNA ligation buffer (Thermo) containing 0.5 units $/ \mu 1 \mathrm{~T} 4$ polynucleotide kinase (NEB) and incubated at $37^{\circ} \mathrm{C}$ for $30 \mathrm{~min}$. The $200-\mu 1$ reaction complexes were added to $300 \mu \mathrm{l}$ of $1 \times$ T4 DNA ligation buffer (Thermo) containing 0.5 units/ $\mu 1 \mathrm{~T} 4 \mathrm{DNA}$ ligase (Thermo). Ligation was performed at $20{ }^{\circ} \mathrm{C}$ for $2 \mathrm{~h}$ with rotation at 15 r.p.m. The nuclei were centrifuged at $4{ }^{\circ} \mathrm{C}$ for $5 \mathrm{~min}$ at 1,000 r.p.m. and resuspended in $400 \mu \mathrm{l}$ of $\mathrm{ddH}_{2} \mathrm{O}$. Protein digestion was performed by adding $25 \mu \mathrm{l}$ of $10 \mathrm{mg} / \mathrm{ml}$ proteinase $\mathrm{K}$ 
(Sigma), $50 \mu \mathrm{l}$ of $10 \% \mathrm{SDS}$, and $25 \mu \mathrm{l}$ of $5 \mathrm{M} \mathrm{NaCl}$, and the tubes were incubated for $2 \mathrm{~h}$ at $65{ }^{\circ} \mathrm{C}$. After incubation, an equal volume of phenol:chloroform:isoamyl alcohol (25:24:1) was added to the sample, shaken vigorously, and then centrifuged for $10 \mathrm{~min}$ at 14,000 r.p.m. Next, the supernatant was transferred to a new tube. This process was repeated twice. DNA was precipitated at room temperature with $5 \mu 1$ of Dr. GenTLE Precipitation Carrier (Takara), $50 \mu 1$ of $3 \mathrm{M}$ sodium acetate ( $\mathrm{pH} 5.2$ ), and $555 \mu \mathrm{l}$ of isopropanol. The precipitated DNA was washed once with $80 \%$ ethanol and dissolved in $160 \mu \mathrm{l}$ of $\mathrm{ddH}_{2} \mathrm{O}$. A total of $20 \mu \mathrm{l}$ of $10 \times$ CutSmart buffer, $10 \mu \mathrm{l}$ of SAM (NEB), and $10 \mu \mathrm{l}$ of MmeI (NEB; 2 units/ $\mu \mathrm{l})$ was added to the $160-\mu 1$ DNA sample, and digestion was performed at $37^{\circ} \mathrm{C}$ for $1 \mathrm{~h}$. The digested DNA sample was subjected to electrophoresis in native PAGE gels. The specific 80-bp DLO Hi-C DNA fragments were excised and transferred to a $0.6-\mathrm{ml}$ tube with a pierced bottom. This tube was then placed into a $1.5-\mathrm{ml}$ tube, and the gel slices were shredded by centrifugation at 14,000 r.p.m. for $10 \mathrm{~min}$. A total of $400 \mu \mathrm{l}$ of TE buffer was added to the $1.5-\mathrm{ml}$ tube (to ensure that the shredded gel was fully immersed in buffer), and the mixture was incubated for $20 \mathrm{~min}$ at $-80{ }^{\circ} \mathrm{C}$, followed by a 2-h incubation at $37^{\circ} \mathrm{C}$ with rotation at 15 r.p.m. Next, the shredded gel, along with the buffer, was transferred into the filter cup of a 2ml Spin-X tube filter (Costar, 8160). After centrifugation, the eluate was transferred into a new 2-

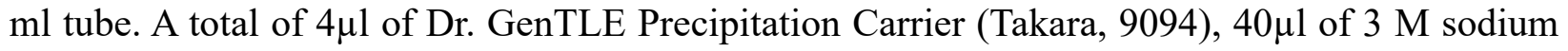
acetate ( $\mathrm{pH}$ 5.2), and an equal volume of isopropanol was added to precipitate the DNA. The precipitated DNA was washed once with $80 \%$ ethanol and dissolved in $40 \mu \mathrm{lof} \mathrm{ddH}_{2} \mathrm{O}$. Next, 1.5

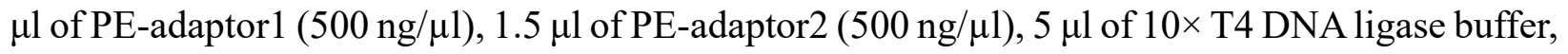
and $2 \mu \mathrm{l}$ of T4 DNA ligase (Thermo, EL0012) were added to the $40 \mu \mathrm{l}$ of DLO Hi-C DNA fragments and incubated at $16^{\circ} \mathrm{C}$ for approximately $30 \mathrm{~min} .90 \mu \mathrm{l}$ of AMPure XP beads (Beckman, A63880) was added to the ligation mixes and washed twice with $80 \%$ ethanol to remove excess Illumina sequencing adaptors. Next, $45 \mu \mathrm{l}$ of $\mathrm{ddH}_{2} \mathrm{O}$ was used to wash DNA from the beads. The eluted DNA was repaired using PreCR Repair Mix (NEB, M0309S) for 20 min at $37{ }^{\circ} \mathrm{C}$ in a final volume of $50 \mu \mathrm{l}$. 5-10 $\mu \mathrm{l}$ of repaired DNA was used as a template and amplified for fewer than 13 cycles. The PCR product is the final DLO Hi-C sequencing library. 


\section{Single cell DLO Hi-C experiment}

\section{Nuclei preparation}

Five million cells were cross-linked with $1 \%$ formaldehyde (Sigma) for 10 mins, and lysed in lysis buffer $\left(10 \mathrm{mM}\right.$ Tris- $\mathrm{HCl}\left(\mathrm{pH} 8.0\right.$ at $\left.25^{\circ} \mathrm{C}\right), 10 \mathrm{mM} \mathrm{NaCl}, 0.3 \%$ Igepal CA-630, 0.5\% SDS, and complete protease inhibitor (Roche)) at $60{ }^{\circ} \mathrm{C}$ for $5 \mathrm{~min}$, and placed on ice immediately. After incubation, the nuclei were pelleted by centrifugation at 1,000 r.p.m. for 5 min and washed once with nuclei wash buffer (PBS which contain $0.5 \%$ Triton X-100, 0.05\% Tween 20, and 0.05\% CA630).

\section{MseI digestion}

The digestion buffer (a total of $310 \mu \mathrm{l}$ of $\mathrm{ddH}_{2} \mathrm{O}, 20 \mu \mathrm{l}$ of $20 \%$ Triton X-100, $40 \mu \mathrm{l}$ of $10 \times$ NEBuffer 2.1, and $30 \mu \mathrm{l}$ of MseI (NEB, 10 units/ $\mu \mathrm{l})$ ) was then added to the nuclei pellet and digested for $3 \mathrm{~h}$ at $37^{\circ} \mathrm{C}$ in thermomixer (Eppendorf) with rotation at 1000 r.p.m.

\section{Indexed half linker ligation}

After digestion, $560 \mu 12.1 x$ T4 DNA ligase buffer were added to the nuclei, and divided into 96well plates and adjusted to the volume of $10 \mu \mathrm{l} /$ tube. Next, $1 \mu \mathrm{l}$ barcoded half linkers $(50 \mu \mathrm{M} / \mu \mathrm{l})$

(Supplementary Table 8) were added to each tube and mixed well. After incubation at room temperature for $5 \mathrm{~min}, 1 \mu \mathrm{T} 4 \mathrm{DNA}$ ligase (Thermo, 5 units/ $\mu \mathrm{l}$ ) were added to each tube. The ligation reaction was performed at $20^{\circ} \mathrm{C}$ for $30 \mathrm{mins}$, and then incubated for 10 minutes at $4{ }^{\circ} \mathrm{C}$. The sample in 96-well plates were transferred to $1.5 \mathrm{ml}$ DNA LoBind Tube (Eppendorf, B148089M), centrifuged at 1,000 r.p.m. for 5 min in $4{ }^{\circ} \mathrm{C}$, and washed 4 times with nuclei wash buffer.

\section{Fragment-end phosphorylation and in situ proximity ligation}

The linker-ligated nuclei were gently resuspended in $200 \mu \mathrm{l}$ of $1 \times$ T4 DNA ligation buffer (Thermo) containing 0.5 units/ $\mu 1 \mathrm{~T} 4$ polynucleotide kinase (NEB) and then incubated at $37{ }^{\circ} \mathrm{C}$ for $30 \mathrm{~min}$. The $200-\mu 1$ reaction complexes were added to $300 \mu 1$ of $1 \times$ T4 DNA ligation buffer (Thermo) containing 0.5 units/ $\mu 1 \mathrm{~T} 4 \mathrm{DNA}$ ligase (Thermo). Ligation was performed at $20{ }^{\circ} \mathrm{C}$ for $2 \mathrm{~h}$ with rotation at 15 r.p.m. The nuclei were centrifuged at $4{ }^{\circ} \mathrm{C}$ for 5 min at 1,000 r.p.m. and resuspended 
in nuclei wash buffer.

\section{Single cell selection and multiple displacement amplification}

The nuclei were stained by DAPI (Thermo) and diluted by nuclei wash buffer. The density of nucleus in liquid was carefully checked under the fluorescence microscope, and adjust to 30 nuclei/ $\mu$ l. Next, about $1 \mu \mathrm{l}$ nuclei were digested with proteinase $\mathrm{K}$ to release the DNA, the reaction

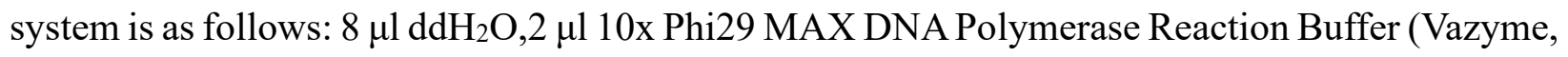
N106), $5 \mu \mathrm{l} 5 \mathrm{~N}$ random primer $(100 \mu \mathrm{M}), 2 \mu \mathrm{dNTP}(10 \mathrm{mM}$ each $), 1 \mu \mathrm{l}$ nuclei ( 30 nuclei/ $\mu \mathrm{l})$, and $1 \mu \mathrm{l}$ proteinase $\mathrm{K}(20 \mathrm{mg} / \mathrm{ml})$. The reaction was performed at $60{ }^{\circ} \mathrm{C}$ for 1 hour, $98{ }^{\circ} \mathrm{C}$ for 10 $\min$, and $4{ }^{\circ} \mathrm{C}$ for $5 \mathrm{~min}$. After proteinase K digestion, $1 \mu 1$ Phi29 MAX DNA Polymerase (Vazyme, N106) was added to the sample, mixed well and incubated in $30^{\circ} \mathrm{C}$ for 3 hours.

\section{Multiple displacement amplification recycle and sequencing library construction}

After multiple displacement amplification (MDA) reaction, $1 \mu 1$ 10\% SDS and $79 \mu \mathrm{ldd} \mathrm{d}_{2} \mathrm{O}$ were added to the sample, and the DNA was purified by $200 \mu \mathrm{l}$ VAHTS DNA Clean Beads (Vazyme, N411-01). The following steps of MmeI digestion, 80bp contact DNA fragment recovery are the same as in situ DLO Hi-C protocol described above. For sequencing library construction, we replaced the previous Illumina sequencing adapter with homemade MGI-2000 platform sequencing adapter with a second round of indexes (Supplementary information, Fig. S3c and Table S8). Previous similar methods ${ }^{36,37}$ have shown that due to the number of barcodes $(96 \times 14)$ far more than the number of nuclei $(30 \times 14)$, therefore most of single nuclei are labeled by a unique barcodes.

\section{DNA-FISH}

The primary hybridization probes contain target sequence and readout sequence ${ }^{38}$. Different groups of probes containing $70 \mathrm{nt}$ target sequences complementary to the genomic region of interest (around $50 \mathrm{~kb}$ ) were designed using OligoMiner software ${ }^{39}$. Each group of probes shared a unique 25-nt readout sequence complementary to the readout probes. All the probes contain more than 18-nt homology to the repetitive sequences from Repbase were filtered out for specificity ${ }^{40}$. 
649

650

651

652

653

654

655

656

657

658

659

660

661

662

663

664

665

666

667

668

669

670

671

672

673

674

675

The primary probe loci for GBP2 is chr1:89320076-89370076, and for GBP5 is chr1:8981787489867874.

DNA FISH was performed following Fast FISH protocol with a little modifications ${ }^{41}$. The cells were fixed with $4 \%$ formaldehyde solution (Sigma, 47608-250ML-F) at room temperature for 5 min followed by PBS buffer washing for three times. Cell membrane and nucleus membrane were permeabilized by methanol incubation for $5 \mathrm{~min}$ followed by PBS buffer washing. Next, the cells were incubated in $0.1 \mathrm{M}$ HCL for 5 mins. After incubation, the cells were heated on a hot plate at $82{ }^{\circ} \mathrm{C}$ for $10 \mathrm{~min}$ in $80 \%$ formamide (Sigma) and $2 \times \mathrm{SSC}$ for DNA denaturation. After denaturation, cells were incubated for 12 hours in hybridization solution with $2 \mu \mathrm{M}$ primary hybridization probes in the presence of $50 \%$ formamide, $8 \%$ dextran sulfate sodium salt (Sigma), and $2 \times$ SSC followed by PBS buffer washing for three times. The cells were then incubated for 30 min in a second hybridization solution with $1 \mu \mathrm{M}$ readout probes in the presence of $30 \%$ formamide and $2 \times \mathrm{SSC}$ followed by PBS buffer washing for three times and $30 \%$ formamide and $2 \times \mathrm{SSC}$ washing for 3 times. Next, the slides were stained with DAPI (Life Technologies) and observed under super-resolution microscope (Nikon, N-SIM).

\section{Immunofluorescence combined with DNA-FISH}

Cells grown on coated glass were fixed in $4 \%$ formaldehyde solution (Sigma, 47608-250MLF) at room temperature for 5 min followed by PBS buffer washing for three times. Next, cells were permeabilized with $0.4 \%$ SDS in PBS for 5 min at RT. Following three washes in PBS for 5 min, cells was blocked in blocking buffer (1× PBS/ 5\% normal serum / 0.3\% Triton X-100) for 60 min. After blocking, antibodies (anti-BRD4, Abcam ab128874; anti-MED1, Abcam ab64965) in antibody dilution buffer $(1 \times$ PBS / 1\% BSA / 0.3\% Triton X-100) at $2 \mu \mathrm{g} / \mathrm{ml}$ final concentration were incubated with the slides overnight at $4{ }^{\circ} \mathrm{C}$. Slides were then washed three times with PBS and recognized by secondary antibodies (Goat antiRabbit IgG Alexa Fluor 488, Life Technologies
A11008, 1:1000 dilution) in the dark for $30 \mathrm{~min}$.

After immunofluorescence, cells were placed in prewarmed PBS and incubated at $60{ }^{\circ} \mathrm{C}$ for 
676

677

678

679

680

681

682

683

684

685

686

687

688

689

690

691

692

693

694

695

696

697

698

699

700

701

702

$20 \mathrm{~min}$, then incubated in 70\% ethanol, $85 \%$ ethanol and then $100 \%$ ethanol for $1 \mathrm{~min}$ at RT. After alcohol dehydration, the cells were heated on a hot plate at $82{ }^{\circ} \mathrm{C}$ for $10 \mathrm{~min}$ in $80 \%$ formamide (Sigma) and $2 \times \mathrm{SSC}$ for DNA denaturation. Next, cells were incubated for 12 hours in hybridization solution with $2 \mu \mathrm{M}$ Alex555-dUTP labeled GBP DNA FISH probes (Chr1: 89,448,890- 89,739,345, Spatial FISH Co. Ltd.) in the presence of $50 \%$ formamide, $8 \%$ dextran sulfate sodium salt (Sigma), and $2 \times \mathrm{SSC}$. After hybridization the cells were washing for three times with $30 \%$ formamide and 3 times with $2 \times$ SSC. Next, the slides were stained with DAPI (Life Technologies) and observed under super-resolution microscope (Nikon, N-SIM).

\section{CFU assays}

Bone-marrow-derived macrophage cells $(\mathrm{BMDM})$ were seeded into 6-well plate $\left(1 \times 10^{5} /\right.$ well $)$ and infected with H37Ra at MOI (multiplicity of infection) 20. Four hour later, the infected cells were washed twice with pre-warmed PBS and incubated with fresh culture medium. Then the cells were treated with AdoCbl (Sigma, Cat\# C0884) at final concentration of $200 \mu \mathrm{M}$. The PBS solution used as a negative control. 3 days later, the cells were lysed with $0.1 \%$ triton X-100 and plated for bacterial burden enumeration using a serial dilution method on plates of Middlebrook 7H11 agar containing OADC enrichment and BBL MGIT PENTA antibiotics (BD, Cat\# 245114). CFUs were counted after 3 to 4 weeks incubation at $37^{\circ} \mathrm{C}$.

\section{Immunofluorescence}

For immunofluorescence of GBP1-5, Rab7, and NF-אB, monocyte derived macrophages (Thp1-macro) were infected with RFP-H37Ra at MOI (multiplicity of infection) 20. The infected cells were washed twice with pre-warmed PBS four hours and incubated with fresh culture medium for another 8 hours. Next, the cells were cross-linked with $4 \%$ formaldehyde for 15 min at room temperature and rinsed three times with $1 \times$ PBS. The specimen was blocked in blocking buffer (1× PBS/ 5\% normal serum / 0.3\% Triton X-100) for 60 min. After blocking, respective antibodies (GBP1-5, Santa Cruz, sc-166960 AF488; Rab7, Santa Cruz, sc-376362 AF488; NF-кb 
p65, Santa Cruz, sc-8008) in antibody dilution buffer (1× PBS / 1\% BSA / 0.3\% Triton X-100) at $2 \mu \mathrm{g} / \mathrm{ml}$ final concentration were incubated with the slides overnight at $4{ }^{\circ} \mathrm{C}$. Slides were washed three times with PBS and stained with DAPI (Life Technologies) and observed under fluorescence microscope.

\section{Mouse Models}

All wild type C57BL/6 mice used in this study were purchased from Beijing Vital River Laboratory Animal Technology and all the experiments in this study were approved by the Scientific Ethic Committee of Huazhong Agricultural University (NO. HZAUMO-2019-019) and maintained at the Laboratory Animal Centre of Huazhong Agriculture University under specific pathogen-free (SPF) conditions with 12-hour light/dark cycles. Based on the principles of laboratory animal welfare and ethics, this study optimized the design of the project and strictly plans the number of animals required. A total of 28 6-week-old female C57BL/6 mice (20 $\pm 2 \mathrm{~g})$ were planned. Among them, 4 were used for bone marrow macrophage isolation experiments, and the remaining 24 were used for Mycobacterium tuberculosis H37Ra infection test and AdoCbl drug treatment experiment.

\section{Animal experiment}

For M.tb infection, 6 week old female mice $(20 \pm 2 \mathrm{~g})$ were infected by intravenous injection with a dose of $5 \times 10^{6} \mathrm{cfu} / \mathrm{mL}$ of $200 \mu \mathrm{L}$ M.tb H37Ra suspension. All animals were randomly distributed into 2 groups of 12 each. Then mice were received a gavage feeding of AdoCbl (Sigma, Cat\# C0884) at a daily dose of $0.5 \mathrm{mg} / \mathrm{kg} \cdot \mathrm{bw}$ and equal volume of sterile PBS used as a negative control. These mice were given the therapy for 1 day post of $M$.tb infection. Mice were euthanized after 15 days of drug treatment. Lungs and spleen were taken out for histopathological observation and CFU analysis. A part of left lung was fixed in 4\% paraformaldehyde for a minimum time of 48h, and tissues were paraffin embedded to make section for haematoxylin and eosin (H\&E) staining. Homogenates of lungs and spleen were plated for bacterial burden enumeration using a 
serial dilution method on plates of Middlebrook 7H11 agar containing OADC enrichment and BBL MGIT PENTA antibiotics (BD, Cat\# 245114) for inhibition of contamination. CFUs were counted after 3 to 4 weeks incubation at $37^{\circ} \mathrm{C}$.

\section{RNA-Seq analysis}

FastQC (version: v0.11.8) was used to assess the quality of RNA-Seq reads, and Trimmomatic ${ }^{42}$ (version: 0.33 ) was used to filter out the low quality bases and adapter sequences. Clean reads longer than $36 \mathrm{bp}$ at both ends were kept for further processing. TopHat ${ }^{43}$ (version: v2.1.1) with bowtie $2^{44}$ (version: 2.3.5.1) was used to align the paired end RNA-Seq reads to human reference genome (hg19) with transcriptome annotations from Ensembl ${ }^{45}$. HTSeq ${ }^{46}$ (version: 0.11.2) was used to count the mapped reads on the transcripts corresponding to each gene. DESeq $2{ }^{47}$ was used for normalization and differential expression analysis with the read counts on the genes as inputs.

\section{ATAC-Seq analysis}

The reads filtering steps were the same as in "RNA-Seq analysis". Trimmed reads were aligned to the human genome assembly (hg19) with the Burrows-Wheeler Aligner-MEM ${ }^{48}$ (version: 0.7.17-r1188). For each sample, SAMtools ${ }^{49}$ (version: 1.7) was used to sort the mapped reads by position. After marking the duplication reads by Picard toolkit (version: 1.119), uniquely mapped reads and non-redundant sequences were kept for further analysis by SAMtools with the parameter F: 1024, q 20. F-Seq ${ }^{50}$ (version: 3) was used to call ATAC-Seq peaks as ENCODE ATAC-Seq data analysis pipeline, and the top 100,000 accessible regions for all samples were kept further analysis.

\section{ChIP-Seq analysis}

The reads filtering steps were the same as "RNA-Seq analysis". Burrows-Wheeler Aligner$\mathrm{MEM}^{48}$ (version: 0.7.17-r1188) was applied to map the reads to the human genome assembly (hg19). Mapped reads were sorted by position and duplications were discarded by SAMtools ${ }^{49}$ 
(version: 1.7). The reads with the mapping quality higher than 30 were considered as uniquely mapped reads. Peaks were called by $\mathrm{MACS}^{51}$ (version: 2.1.1.20160309). For broad peaks, the parameters were -B --broad -q 0.05. For narrow peaks, the parameter was -B.

\section{Annotation of chromatin states of enhancer and promoter}

Promoter regions were defined as the genomic regions $1 \mathrm{~kb}$ in front of the transcription start sites (TSS) of RefSeq genes. Repressed promoters were defined as the promoter regions without $\mathrm{H} 3 \mathrm{~K} 4 \mathrm{me} 3$ peaks. Bivalent promoters were defined as the promoter regions enriched with $\mathrm{H} 3 \mathrm{~K} 4 \mathrm{me} 3$ and $\mathrm{H} 3 \mathrm{~K} 27 \mathrm{me} 3$ peaks. Active promoters were defined as the promoter regions enriched with $\mathrm{H} 3 \mathrm{~K} 4 \mathrm{me} 3$ peaks but without $\mathrm{H} 3 \mathrm{~K} 27 \mathrm{me} 3$ peaks.

For each cell type, the open non-promoter regions with ATAC-Seq peaks were initially selected as the candidate regions of enhancers. As previously described ${ }^{52,53}$, the candidate regions with H3K4me1 peaks were defined as enhancers. The active mark H3K27ac and inactive mark H3K27me3 were combined to defined the different state of enhancers. An enhancer with H3K27me3 peaks was defined as poised enhancer. If there were H3K27ac peaks located in enhancer region, the enhancer was defined as active enhancer. The enhancers with neither $\mathrm{H} 3 \mathrm{H} 27 \mathrm{ac}$ nor $\mathrm{H} 3 \mathrm{~K} 27 \mathrm{me} 3$ peaks were considered as primed state.

\section{In situ DLO Hi-C analysis}

As the read1 sequences in the $2 \times 150 \mathrm{bp}$ paired-end reads in the in situ DLO Hi-C library contains all the chromatin interaction pair information, only readl sequences were retained for further analysis. Linker filtering was conducted with a Java program, DLO Hi-C Tool ${ }^{54}$. Reads with mapping scores of greater than 32 were retained for subsequent analysis. Via the linker sequence, the sequences with interaction pairs information were extracted from the raw reads.

To increase the alignment rate, the restriction endonuclease recognition site was complemented at the end of the sequence. Burrows-Wheeler Aligner-ALN ${ }^{48}$ (version: 0.7.17-r1188) was used to align the interaction sequences to the human reference genome (hg19) with the parameter $-\mathrm{n} 0$. 
Only the uniquely mapped reads with mapping quality (MAPQ) scores of $\geqslant 20$ were retained and paired for further analysis.

The reference genome was divided into fragments according to the restriction enzyme sites, and the uniquely mapped sequence pairs were aligned to the restriction enzyme fragments. If two ends from a paired-read were mapped to the same restriction enzyme fragment, the paired-read was considered a self-ligation product. If two ends from a paired-read were mapped to two adjacent restriction enzyme fragments, the paired-read was considered a religation product. Both the selfligation and religation reads were excluded for further analysis. If multiple sequences had both ends aligned to the same positions, only one sequence was retained for further analysis, since such reads probably resulted from PCR amplification.

To identify chromatin loops, interaction matrices were converted to hic files by the pre command of Juicer Tools ${ }^{55}$ with default resolutions. The HiCCUPS algorithm of Juicer Tools was used to generate loop lists at resolutions of $5 \mathrm{~kb}, 10 \mathrm{~kb}$ and $25 \mathrm{~kb}$.

\section{Single cell DLO Hi-C data analysis}

The processing of the sciDLO Hi-C sequencing data are basically as same as the bulk DLO Hi-C data processing ${ }^{54}$, with modifications in the PETs extraction step to recognize the barcodes and an additional step to split the final valid reads according to the barcodes. These two additional steps were implemented in a new sciDLO Hi-C package.

In PETs extraction step, the parameter "--fq1 [R1_file] --fq2 [R2-file] -linker GTCGGANNNNNNNNGCTAGCNNNNNNNNTCCGAC --enzyme $\mathrm{T}^{\wedge} \mathrm{TA} \wedge \mathrm{A}^{\prime}$ were used. For cell split step, the default parameters were used: 1, 2, 4 differences of hamming distance were allowed for the comparison between "read barcode VS library barcode", "barcode1 VS barcode2 (within same reads)", "barcode R1 VS barcode R2 (barcode within R1 and R2)."

Single cell embedding using the scHiCTools package ${ }^{23}$, all cells matrix was generated with 1 $\mathrm{Mb}$ resolution. For cell embedding using the "InnerProduct" to calculate the similarity between all cells, and using MDS method to mapping all cell's contact matrix to a 2 dimension vector. Thp- 
811 M.tb cells are classified into "immune highly activated" and "immune lowly activated" by the 812 threshold of the mean value of all Thp-M.tb cell's "separation score", which equal to the mean 813 distance to the closest 4 Thp1-macro cells in the 2 dimension space.

814 To calculate local DoD from single cell data, the bulk cell DoD calculation pipeline was used 815 with a few modifications including: 1) merged single cell data as input; 2) the "significant interaction points" in the first step was replaced by the single cell long-range ( $>20 \mathrm{~kb})$ contacts; 3 )

817 the mean distance was divided by $5 \mathrm{~kb}$, to scale at the same level as the bulk cell DoD value; 4) 818 sampling the same number of the contacts in the compared regions of different samples when DoD comparison between samples was performed.

\section{Simulation of chromosome three-dimensional structure of single cells}

The chromatin were simulated to generate the chromatin 3D structure using software "nuc_dynamic" 56 . For global chromosomes simulation, the software was run with the parameter: “-s 8.0 4.0 2.0 1.0 0.4 0.2 0.1". For local region structure simulation, the parameter "-s 0.10 .05 0.010 .005 " was used. And the results were saved as Protein Data Bank (PDB) file format and visualized with PyMOL software (version: 2.3.0).

\section{Hi-C loop analysis}

The Thp1-mono and Thp1-macro contact matrices were randomly downsampled to $351,514,529$ intrachromosomal contacts (the same number of intrachromosomal contacts as the Thp1-M.tb matrix). The HiCCUPS algorithm was employed to call loops from the normalized Thp1-mono and Thp1-macro libraries. To find the differential interaction loops between two samples, the loops were divided into overlapping and nonoverlapping loops. For differential analysis of overlapping loops, the surrounding $5 \times 5$ window was compared between the two matrices by Wilcoxon tests, as previously described ${ }^{57}$. Loops with p-values of less than 0.05 were considered significantly different. For nonoverlapping loops, the fold enrichment values of the peak over all local neighborhoods were also calculated in the sample without the loop, as 
838

839

840

841

842

843

844

845

846

847

848

849

850

851

852

853

854

855

856

857

858

859

860

861

862

863

864

previously described $^{58}$. If all calculated fold enrichment values of the pixels in the sample without the loop were lower than 1.3, the peak in the sample with the loop was considered a differential peak.

\section{TAD boundary calling}

TAD boundaries were called at a resolution of $40 \mathrm{~kb}$, as previously described ${ }^{59}$. The interaction frequencies within $2 \mathrm{Mb}$ downstream and $2 \mathrm{Mb}$ upstream were compared with the default parameters of DomainCaller. The directionality index (DI) was used to quantify the bias in each bin. Domains were inferred from hidden Markov model (HMM) state calls.

If the distance between two TAD boundaries in two samples was within $80 \mathrm{~kb}$, these TAD boundaries were considered conserved TAD boundaries in the two samples. If the distance between two TAD boundaries was greater than $80 \mathrm{~kb}$, the two TADs were considered shifted TADs ${ }^{60}$. If one TAD in the previous cell state corresponded to multiple smaller TADs in the sample of the subsequent cell state, the TAD was considered a separated TAD. If multiple small TADs in the previous cell state corresponded to a large TAD in the subsequent cell state, this TAD was considered a fused TAD.

The hiclib library was applied to distinguish A and B compartments as previously described ${ }^{61}$. If the values in the first eigenvector were higher than 0 , the corresponding bins were marked as $\mathrm{A}$ compartments. If the values in the first eigenvector were smaller than 0 , the corresponding bins were marked as B compartments.

\section{TAD "Degree of Disorder" (DoD) and Co-Regulation Score (CRS) calculation}

The significant chromatin interactions were first identified based on a Poisson process model.

The expected value was calculated by considering both distance-dependent decay and the local interaction background, as described in previous studies ${ }^{58,62}$. The window parameters $p$ and $w$ were set to 4 and 7, respectively, according to previous studies ${ }^{58,62}$. After allocating all significant interactions in the TAD contact matrix $M$, the $\mathrm{k}$ nearest neighbors $P k_{i}$ with $\mathrm{k}=3$ for each 
significant interaction $p_{i}=\left(x_{i}, y_{i}\right), i \in[1, n]$ were calculated. The mean distance within contact matrix $\left(M D_{i}\right)$ between the significant point $p_{i}$ was measured as the mean distance to its k nearest neighbors, as shown in Supplementary information, Fig. S2f:

$M D_{i}=\frac{\sum \operatorname{dist}\left(p_{i}, p_{j}\right)}{k}$

where $\operatorname{dist}\left(\mathrm{p}_{\mathrm{i}}, \mathrm{p}_{\mathrm{j}}\right)=\sqrt{\left(\mathrm{x}_{\mathrm{j}}-\mathrm{x}_{\mathrm{i}}\right)^{2}+\left(\mathrm{y}_{\mathrm{j}}-\mathrm{y}_{\mathrm{i}}\right)^{2}}$ is the Euclidean distance in the matrix heatmap between $p_{i}$ and $p_{j}$. The KDTree data structure was used to accelerate the nearest neighbors searching process ${ }^{63}$. The mean value of all local $M D s$, DoD $=\sum M D_{\mathrm{i}} / \mathrm{n}$, in a TAD, which represented the "disorder state" of the chromatin interactions within the TAD was defined as the TAD DoD.

Since the two-sample Kolmogorov-Smirnov (KS) test is sensitive to differences in both the location and the shape of the distribution, it was used for statistical comparisons between the TAD DoD values of two biological samples. Here, the D statistic was calculated as follows:

$D_{n, m}=\sup _{x}\left|F_{1, n}(x)-F_{2, m}(x)\right|$

where sup is the supremum function and $F_{1, n}$ and $F_{2, m}$ are the cumulative distribution functions of the $M D$ of different samples. For balancing the effect of sequencing depth, all sample's contact matrices are reconstructed using the same number of valid reads.

The consistency of the overall transcriptional change direction (upregulation or downregulation) of the genes within a TAD during differentiation and activation was defined as the TAD CRS. The regulation direction score (DS) of a TAD $t$ was defined as follows:

$$
\mathrm{DS}_{\mathrm{t}}=\frac{\sum_{\mathrm{i} \in \mathrm{t}} \operatorname{sgn}\left(\log _{2}\left(\text { FoldChange }_{\mathrm{i}}\right)\right)}{\mathrm{N}_{\mathrm{t}}}
$$

where sgn is the sign function. The TAD CRS was defined as the absolute value of the DS: $\mathrm{CRS}_{\mathrm{t}}=\operatorname{abs}\left(\mathrm{DS}_{\mathrm{t}}\right)$

\section{Collection of GWAS-associated risk SNPS}

The TB risk SNPs were obtained from all TB-related GWAS studies. We also obtained two 
extra GWAS results from the GWAS catalog and UK Biobank GWAS data (Supplementary Table 7). The GWAS catalog, which was developed by the National Human Genome Research Institute (NHGRI) and European Molecular Biology Laboratory's European Bioinformatics Institute (EMBL-EBI), contains 3,923 publications and 134,705 SNP-trait associations ${ }^{64}$. Neale Lab released a large body of summary statistics from GWAS analyses on its website ${ }^{65}$, including data for 361,194 individuals from the UK Biobank ${ }^{66}$ (194,174 females and 167,020 males). All GWAS results were filtered with a cutoff $\mathrm{p}$-value of $<1 \times 10^{-5}$ and minor allele frequency (MAF) of $>0.05$ and are listed in (Supplementary Table 7). The p-values for meta-analyses were not adjusted because the raw GWAS data of several previous GWAS analyses were not available.

\section{Integrated analysis of GWAS, eQTL and Hi-C data}

Significant eQTLs associated with risk SNPs were obtained from the GTEx portal, which includes gene expression data for 48 different tissues ${ }^{67}$. The significant genes associated with TB risk SNPs are listed in Supplementary Table 7. Additionally, chromatin interaction loops with risk SNPs overlapped with significant eQTL gene regions within relevant tissues were selected for further investigation and are listed in Supplementary Table 7. The R package ggbio was employed to demonstrate the interactions between SNPs and significant gene regions with Hi-C $\operatorname{loops}^{68}$.

\section{DATA AND SOFTWARE AVAILABILITY}

The accession number for all sequencing data analyzed in this study have been deposited in the Gene Expression Omnibus (GEO) under accession GSE143984 and GSE159501. GWAS catalog, https:/www.ebi.ac.uk/gwas. UK Biobank GWAS result, http://www.nealelab.is/ukbiobank. GTEx, http://www.gtexportal.org. ENCODE ATAC-Seq data analysis pipeline, https://www.encodeproject.org/documents/0eb389f9-d23d-4053-b25b-

1e2826ee5a86/@@download/attachment/ATACpipelineV7.pdf._ Picard toolkit, http://broadinstitute.github.io/picard/. The sciDLO Hi-C data analysis pipeline is available at 
917 https:/github.com/GangCaoLab/sciDLO. The script for DoD calculation is available at 918 https://github.com/GangCaoLab/MDkNN. The ChIP-seq data of NFkB is available at 919 http://hgdownload.cse.ucsc.edu/goldenPath/hg19/encodeDCC/wgEncodeSydhTfbs/. The ChIP920 seq data of MED1 and BRD4 is available at 921 https://www.ncbi.nlm.nih.gov/geo/query/acc.cgi?acc=GSE160670.

922

923

924 


\section{References}

926 36. Cusanovich, D.A. et al. Multiplex single-cell profiling of chromatin accessibility by combinatorial cellular indexing. Science 348, 910-914 (2015).

37. Ramani, V. et al. Massively multiplex single-cell Hi-C. Nature methods 14, 263-266 (2017).

38. Bintu, B. et al. Super-resolution chromatin tracing reveals domains and cooperative interactions in single cells. Science 362, eaau1783 (2018).

39. Beliveau, B.J. et al. OligoMiner provides a rapid, flexible environment for the design of genome-scale oligonucleotide in situ hybridization probes. Proceedings of the National Academy of Sciences of the United States of America 115 (2018).

40. Jurka, J. et al. Repbase Update, a database of eukaryotic repetitive elements. Cytogenetic and genome research 110, 462-467 (2005).

41. Guan, J., Liu, H., Shi, X., Feng, S. \& Huang, B. Tracking multiple genomic elements using correlative CRISPR imaging and sequential DNA FISH. Biophysical journal 112, 1077 1084 (2017).

42. Bolger, A.M., Lohse, M. \& Usadel, B. Trimmomatic: a flexible trimmer for Illumina sequence data. Bioinformatics 30, 2114-2120 (2014).

43. Trapnell, C., Pachter, L. \& Salzberg, S.L. TopHat: discovering splice junctions with RNASeq. Bioinformatics 25, 1105-1111 (2009). 9, 357 (2012). 
945 45. Clamp, M. et al. Ensembl 2002: accommodating comparative genomics. Nucleic Acids $946 \quad$ Research 31, 38-42 (2003).

947 46. Anders, S., Pyl, P.T. \& Huber, W. HTSeq-a Python framework to work with high948 throughput sequencing data. Bioinformatics 31, 166-169 (2015).

949 47. Love, M.I., Huber, W. \& Anders, S. Moderated estimation of fold change and dispersion 950 for RNA-seq data with DESeq2. Genome biology 15, 550 (2014).

951 48. Li, H. \& Durbin, R. Fast and accurate long-read alignment with Burrows-Wheeler 952 transform. Bioinformatics 26, 589-595 (2010).

953 49. Li, H. et al. The sequence alignment/map format and SAMtools. Bioinformatics 25, 2078954 2079 (2009).

955 50. Boyle, A.P., Guinney JCrawford, G.E. \& Furey, T.S. F-Seq: a feature density estimator for 956 high-throughput sequence tags. Bioinformatics 24, 2537-2538 (2008).

957 51. Zhang, Y. et al. Model-based Analysis of ChIP-Seq (MACS). Genome biology 9, R137 958 (2008).

959 52. Chen, H. et al. A pan-cancer analysis of enhancer expression in nearly 9000 patient samples. Cell 173, 386-399.e312 (2018).

961 53. Minoux, M. et al. Gene bivalency at polycomb domains regulates cranial neural crest 962 positional identity. Science 355, eaal2913 (2017).

963 54. Hong, P. et al. The DLO Hi-C Tool for Digestion-Ligation-Only Hi-C Chromosome 964 Conformation Capture Data Analysis. Genes 11, 289 (2020). 
55. Durand, N.C. et al. Juicer provides a one-click system for analyzing loop-resolution Hi-C experiments. Cell Syst. 3, 95-98 (2016).

56. Stevens, T.J. et al. 3D structures of individual mammalian genomes studied by single-cell Hi-C. Nature 544, 59-64 (2017).

57. Kim, Y.H. et al. Rev-erb $\alpha$ dynamically modulates chromatin looping to control circadian gene transcription. Science 359, 1274-1277 (2018).

58. Rao, S.S. et al. A 3D map of the human genome at kilobase resolution reveals principles of chromatin looping. Cell 159, 1665-1680 (2014).

59. Dixon, J.R. et al. Topological domains in mammalian genomes identified by analysis of chromatin interactions. Nature 485, 376-380 (2012).

60. Zirkel, A. et al. HMGB2 loss upon senescence entry disrupts genomic organization and induces CTCF clustering across cell types. Mol. Cell 70, 730-744.e736 (2018).

61. Maxim, I. et al. Iterative correction of Hi-C data reveals hallmarks of chromosome organization. Nat. Methods 9, 999-1003 (2012).

62. Wang, X.-T., Dong, P.-F., Zhang, H.-Y. \& Peng, C. Structural heterogeneity and functional diversity of topologically associating domains in mammalian genomes. Nucleic Acids Res. 43, 7237-7246 (2015).

63. Bentley, J.L. Multidimensional binary search trees used for associative searching. Commun. ACM 18, 509-517 (1975).

64. MacArthur, J. et al. The new NHGRI-EBI catalog of published genome-wide association 
studies (GWAS catalog). Nucleic Acids Res. 45, D896-D901 (2016).

986 65. Bycroft, C. et al. The UK Biobank resource with deep phenotyping and genomic data. Nature 562, 203-209 (2018).

988 66. Sudlow, C. et al. UK Biobank: an open access resource for identifying the causes of a wide range of complex diseases of middle and old age. PLoS Med. 12, e1001779 (2015).

990 67. Lonsdale, J. et al. The genotype-tissue expression (GTEx) project. Nat. Genet. 45, 580$585(2013)$.

992 68. Yin, T., Cook, D. \& Lawrence, M. ggbio: an R package for extending the grammar of graphics for genomic data. Genome Biol. 13, R77 (2012). 
995 Acknowledgements

996 This work was supported by the National Key Research and Development Project of China 997 (grant 2017YFD0500300 and 2016YFA0102500 to G.C.), the National Natural Science

998 Foundation of China (grants 31970590 and 31771402 to G.L., 31872470 to G.C., and 31900432

999 to D.L.), Fundamental Research Funds for the Central Universities (grants 2662017PY116 to

1000 G.L., and 2662018PY025 and 2662019YJ004, to G.C.), National Postdoctoral Program for 1001 Innovative Talents (grant BX20180113, to D.L.), China Postdoctoral Science Foundation (grant 1002 2018M640710, to D.L.)

1003

1004 Author contributions

1005 G.C., G.L., and D.L. contributed with conception of the project and experiment design. D.L., Z.Z., 1006 S.Z., L.X., Q.X., J.W., Y.H., X.W., C.C., and Z.C conducted the in situ DLO Hi-C, sciDLO Hi-C, 1007 ChIP-Seq, ATAC-Seq, RNA-seq, Immunofluorescence, DNA-FISH experiments and generated 1008 data. D.L., B.Y., W.Z., Z.H., and R.Y. conducted CRISPR knocked out experiments. W.X., P.H., 1009 C.W., C.W., J.M., and X.H. performed data analysis and interpretation. D.L., G.C., G.L., W.X., 1010 P.H., and C.W. wrote the manuscript, with input from all other authors. Z.F. revised the manuscript. 1011 G.C., and G.L. supervise the project.

1012

1013 Conflict of Interest

1014 The authors declare no competing interests.

1015

1016

1017

1018

1019

1020 


\section{Figure and table legends}

1022

1023

1024

1025

1026

1027

1028

1029

1030

1031

1032

1033

1034

1035

1036

1037

1038

1039

1040

1041

1042

1043

1044

1045

1046

1047

Fig. 1 | Decoding the 3D Genome of monocytes, macrophages, and M.tb infected macrophages with single cell indexed (sci) DLO Hi-C. a, Representative calcein and hoechst fluorescence staining images of Thp1-mono, Thp1-macro, and Thp1-M.tb cells. b, Flowchart of the sciDLO Hi$\mathrm{C}$ method. c, Present cluster analysis result of sciDLO Hi-C data sets by using two-dimensional scatter plots. d, Comparison of average contacts around immune genes ( $\pm 10 \mathrm{~Kb}$ around TSS sites) between Thp1-mono, Thp1-macro, and Thp1-M.tb cells. e, Simulation of chromatin threedimensional conformation of representative immune highly activated and moderately activated cells. f, Comparison of the spatial location of NOD2 and BRD7 in immune highly activated and moderately activated cells by using simulated nucleus. NOD2 and BRD were marked with arrows.

Fig. 2 | Evaluating the order and stochasticity of single cell chromatin folding with degree of disorder (DoD). a,b, Bulk cell chromatin contact matrix and gene expression level of STAT1 TAD of Thp1-macro and Thp1-M.tb cells. The chromatin interaction hot spot which formed in low DOD TAD were marked by dashed box. Chromatin loop mediated STAT1 and enhancer interaction were labeled by arrows. c,d, Single cell chromatin contacts around STAT1 gene of Thp1-macro and Thp1-M.tb cells. Individual cells have similar chromatin folding patterns in the low DoD TAD. e, Illustration of the TAD degree of disorder (DoD) which was used to measure the order and consistency of chromatin folding in single cells. f, DoD values of Thp1-mono and Thp1-macro around MYC gene region by using bulk cell DLO Hi-C data sets. g, Relationship between TAD DoD and average gene expression level (normalized read count) in Thp1-mono, Thp1-macro, and Thp1-M.tb, respectively. The DoD value higher than median was defined as "High DoD"; lower than the median was defined as "Low DoD". Significance of differences was measured by Kolmogorov-Smirnov test. $\mathbf{h}$, Correlation between the TAD DoD and A, B compartments. In $\mathrm{x}$ axis, B/A means the TADs which contains both A and B compartments. Significance of differences was measured by unpaired one-sided t test. i, Correlation between the TAD DoD and coregulation score (CRS). TADs with lower TAD DoD preferentially have higher CRS, underlying more 
1048

1049

1050

1051

1052

1053

1054

1055

1056

1057

1058

1059

1060

1061

1062

1063

1064

1065

1066

1067

1068

1069

1070

1071

1072

1073

1074

coordinated gene regulation in such TADs. $\mathrm{x}$ axis is the average value of the quantile groups with the lowest, low, high and highest TAD DoDs, respectively. $\mathbf{j}$, Absolute values of the log2 fold changes in gene expression (y-axis) in the boundary not changed but DoD value significant changed TADs (dynamic DoD), boundary and DoD unchanged TADs (static DoD), boundary shifted TADs (shifted), boundary fused TADs (fused), and boundary divided TADs (divided) during differentiation and infection.

\section{Fig. 3 | Low DoD is associated with active epigenetic modification and high gene expression} level. a,b, Correlation between TAD DoD and chromatin epigenetic profile. The y axis shows the normalized ChIP-seq peaks per TAD. The DoD value higher than median was defined as "High DoD"; lower than the median was defined as "Low DoD". Significant differences were measured by unpaired one-sided t test. c, Correlation between TAD DoD and chromatin accessibility. Each dot represents one TAD. $\mathrm{x}$-axis is the number of ATAC peaks per Mb. d,e, Chromatin contact matrix and DoD value changes around HERC5 and HERC6 gene region after M.tb infection. f,g, RNA expression level, chromatin accessibility, H3K27ac, and H3K4me4 histone modification changes after M.tb infection. h,i, Simulated three-dimensional structure of HERC5 and HERC6 gene region during M.tb infection by using bulk cell DLO Hi-C data sets. j, The relationship between DoD value, chromatin folding order, chromatin accessibility, gene transcription coregulation, and histone modification.

\section{Fig. 4 | Remodeling of the chromatin configuration of guanylate-binding protein (GBP) gene} clusters. a, Comparison of TAD DoD value, chromatin interaction matrices, chromatin loops, RNA expression levels and epigenetic modifications around the GBP loci in bulk cells before and after M.tb infection. b, Comparing the single cell chromatin contact differences using merged single cell chromatin contact matrix. Each color of the dots in the matrix represents the chromatin contact from the same cell. c, Single cell chromatin contacts between $G B P 2, G B P 4, G B P 5$, and GBP7 and RNA expression before and after M.tb infection. d, Depiction Hi-C chromatin loops 
1075 before and after infection and BRD4 and MED1 ChIP-seq peaks around GBP gene clusters. e, Co1076 localization between BRD4 and the GBP gene cluster by IF and DNA-FISH in Thp1-macro and 1077 Thp1-M.tb cells. IF, DNA-FISH, and merged channels (overlapping signal in white) are shown in 1078 separate images. The dashed line highlights the nuclear periphery, determined by DAPI staining. 1079 The rightmost column shows the area in the yellow box in greater detail. Each cell type, we counted 108060 GBP gene loci, 18.3\% (11/60) GBP loci were co-located with BRD4 in Thp1-macro cells and $1081 \quad 65.0 \%$ (39/60) GBP loci were co-located with BRD4 in Thp1-M.tb cells. TAD DoD of THP-1 cells during differentiation and infection. The y-axis represents the average DoD value of TADs in each state. b, The relationship between TAD DoD and chromatin loop during differentiation and activation. The $\mathrm{x}$-axis represents $\log 2$ fold change of TAD DoD value, and the $\mathrm{y}$-axis represent the number of loop changes in each TAD. c, Compare NF- $\mathrm{B}$ enrichment around transcription start site $( \pm 3 \mathrm{~Kb})$ between strengthened loop anchor gene and random control gene. The gene in the heatmap were sorted according to the enrichment intensity of NF- $\kappa \mathrm{B}$. ChIP-

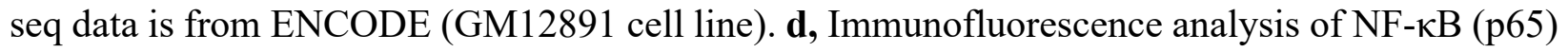
subcellular location before and after M.tb infection. e, Log2 fold changes of ATAC peaks and RNA expression level of NF- $\mathrm{B}$ target genes $(n=435)$ and random control genes. Significant differences were calculated by unpaired one-sided t test. $\mathbf{f}$, The ratio of strengthened loop/total loop of NF-кB target gene sties and random control sites. g, Chromatin loop remodeling and TAD DoD changes in typical NF- $\kappa \mathrm{B}$ target gene loci. $\mathbf{h}$, Bulk cell and merged single cell chromatin interaction matrices around IFIT gene locus. Cells with more consistent chromatin interaction were marked with a dashed line. $\mathbf{i}, \mathbf{j}$, Comparison of chromatin loop configurations, RNA expression levels, and 1098 epigenetic modifications of the IFIT gene family locus before (i) and after (j) M.tb infection. The 1099 arrows in (j) indicate the potential cis element linked with IFIT2, IFIT3, and IFIT1. k, Schematic diagram of chromatin remodeling of $\mathrm{NF}-\kappa \mathrm{B}$ target genes during infection. 
1102

1103

1104

1105

1106

1107

1108

1109

1110

1111

1112

1113

1114

1115

1116

1117

1118

1119

1120

1121

1122

1123

1124

1125

1126

1127

1128

Fig. 6 | Functional identification of PD-L1 enhancer. a, Interaction of the PD-L1 enhancer and promoter in the Hi-C matrix. b,c, Genome Browser view of RNA expression levels, chromatin accessibility, and histone modifications at the PD-L1 gene and enhancer regions in Thp1-macro and Thp1-M.tb cells. NF-кB ChIP-seq peaks (ENCODE, GM15510 cell line) and strengthened ATAC-seq peaks were marked by arrows. d, ChIP-qPCR validation of the NF- $\kappa \mathrm{B}$ (P65) enrichment on the PD-L1 enhancer and promoter regions. The amount of immunoprecipitated DNA in each sample is represented as signal relative to the total amount of input chromatin (y-axis). Significant differences were calculated by unpaired one-sided t test. e, Experimental design of sgRNA-guided enhancer perturbation by the Cas9 protein. f, Relative mRNA expression levels of $P D-L 1$ in Thp1macro cells and the same line after enhancer deletion. Error bars show mean $\pm \mathrm{SE}$ of results of three replicates; the difference was assessed using unpaired one-sided t test. g, PD-L1 protein levels in Thp1-macro cells and the same cell line after enhancer deletion before and after M.tb infection. $\mathbf{h}$, Schematic of enhancer-promoter interaction mediated regulation of PD-L1 expression during M.tb infection.

Fig. 7 | Identification of long range regulatory target genes of TB susceptibility loci and novel anti-TB drug targets by integrated multi-omics analysis. a, Identification of long range regulatory target genes of TB susceptibility loci, LRRK2 by integrated eQTL, Hi-C and GWAS multi-omics analysis. b, The rs 1873613 SNP is located in the anchor of the LRRK2 gene chromatin loop, which is significantly strengthened after M.tb infection, as shown in the Hi-C matrix. c, Simulated typical single cell chromatin structure of SNP rs1873613 and LRRK2 gene region before and after M.tb infection. d, Regulation of LRRK2 gene expression by the rs1873613 SNP. The $\mathrm{x}$ axis indicates three genotypes of individuals from the Genotype-Tissue Expression (GTEx) dataset. e,f, Immunofluorescence analysis and quantification of the co-localization of the M.tb (H37Ra-RFP) and Rab7 in bone marrow-derived macrophages treated with the LRRK2 inhibitor AdoCbl and PBS (control). g, CFU assays of M.tb in bone-marrow-derived macrophage cells treated with AdoCbl and PBS (control). Significant differences were calculated by unpaired one- 
sided t test. h,i, CFU assays of M.tb in C57BL/6 mouse lungs treated with AdoCbl and PBS (control). Significant differences were calculated by unpaired one-sided t test. $\mathbf{j}$, HE-stained lung and spleen sections in the AdoCbl and control (PBS) groups. Obvious lesions in the lungs and spleen are highlighted by the dashed lines.

\section{Extended Data Fig. 1 | Transcription dynamics and three-dimensional genome structure of} single cell of THP-1 cells. a, Clustering of gene expression profiles in THP-1 cells during differentiation and M.tb infection. The heatmap shows the relative expression levels of genes (rows) in each sample (column). b, MA plot for gene differential expression analysis during THP-1 cell differentiation. Important transcription factors related to cell proliferation and differentiation are highlighted. c, MA plot for gene differential expression analysis during M.tb infection. Marker genes and important transcription factors for macrophages and M1 macrophages are highlighted. d, Recovery of 80 bp proximity ligation junction DNA by DNA PAGE gel during sciDLO Hi-C. e, Junction reads between different single cell Hi-C data sets. f, The structures of sequence adapter, $80 \mathrm{bp}$ junction contact DNA and amplification primers used in sciDLO Hi-C experiment. g, Simulated single cell 3D genome structure of Thp1-mono, Thp1-macro, and Thp1-M.tb cells at 25 $\mathrm{kb}$ resolution. $\mathbf{h}$, Identifying chromatin territories by using simulated single cell nucleus. $\mathbf{i}$, Pearson coefficient of merged single cell chromosome 1 contact matrix and bulk cell chromosome 1 contact matrix.

\section{Extended Data Fig. 2 | Calculation of TAD degree of disorder (DoD) in three types of cells. a,} Histone modification, DNase clusters, and transcription factor enrichment in the STAT1 enhancer region. The transcription factors regulating the PD-L1 gene are marked by arrows. Data are from the UCSC Genome browser (http://genome.ucsc.edu). b, Illustration of computational pipeline for determining the TAD DoD. First, the local expectation of chromatin interactions was estimated using the distance decay function and donut filter. Then, the p-value of each point in the contact matrix was calculated and screened using a Poisson process model. The average distance to each 
1156 significant point's k-nearest neighbors was designated as the MD (mean distance). The TAD DoD 1157 was calculated as the average value of the MD of all points. c, Histogram showing the MD 1158 distribution before and after M.tb infection in the GBP region (Chr1:89,360,000-89,920,000). d,

1159 The cumulative histogram used in the KS (Kolmogorov-Smirnov) test for statistical comparison 1160 of the MD distribution in (c); here, the D statistic was equal to 0.261 , and the p-value was equal to $11613.852 \mathrm{e}-8$. e, Examples of TADs with different TAD DoDs. From left to right, the TAD DoD value 1162 is increasing. f, The TAD DoD distribution in all three cell types. The $\mathrm{x}$-axis represents the TAD 1163 DoD value and the $y$-axis represents the kernel density estimation (KDE) value. g, Schematic 1164 illustrating the calculation of gene coregulation score (CRS) in TAD. h, TAD dynamics of THP-1 1165 cells during differentiation and activation. i, DoD dynamics of boundary intact TADs during 1166 differentiation and infection. M.tb infection. a, Chromatin state definitions and histone mark probabilities. Average genome 1171 state of Thp1-mono, Thp1-macro, and Thp1-M.tb cells, respectively. The difference in the 1172 chromatin state in each sample is highlighted with an arrow. b, Definition of enhancer and 1173 promoter states. c, Dynamics of enhancer and promoter states during THP-1 cell differentiation and M.tb infection. d, Relationship between gene expression levels and histone modifications in 1175 promoter regions. Scatterplots correlating H3K27me3 (x axis), H3K4me3, H3K27ac, and 1176 H3K9me3 (y axis) enrichment levels (log2) at the promoter regions (transcription start site (TSS) $1177 \pm 1 \mathrm{~kb})$ in Thp1-mono, Thp1-macro, and Thp1-M.tb cells, respectively. The color codes indicate 1178 RNA $\log _{2}$ fold changes. e, KEGG pathway analysis of the 423 genes whose promoter status 1179 changed from repressive to active after M.tb infection. 
1183 Co-localization between MED1 and the GBP gene cluster by IF and DNA-FISH in Thp1-macro 1184 and Thp1-M.tb cells. IF, DNA-FISH, and merged channels (overlapping signal in white) are shown 1185 in separate images. The dashed line highlights the nuclear periphery, determined by DAPI staining.

1186 The rightmost column shows the area in the yellow box in greater detail. Each cell type, we counted 118760 GBP loci, $15.0 \%$ (9/60) GBP loci were co-located with MED1 in Thp1-macro cells and $51.6 \%$ 1188 (31/60) GBP loci were co-located with MED1 in Thp1-M.tb cells.

Extended Data Fig. 5 | Transcription factor NF-кB is involved in the strengthening of target chromatin loops during M.tb infection. a, $\log 2$ fold changes of overall TAD DoD value of THP1 cells during differentiation and infection. b, Log2 RNA expression fold changes of the immune genes which located in the strengthened (immune gene, $n=229$ ) and weakened (immune gene,

$1194 \mathrm{n}=112$ ) loop anchors. Significant differences were calculated by unpaired one-sided t test. c, 1195 Dynamic immune gene-regulatory element chromatin interaction network during M.tb infection. d, KEGG pathway enrichment analysis of the genes located in strengthened loop anchors. e, ChIP-

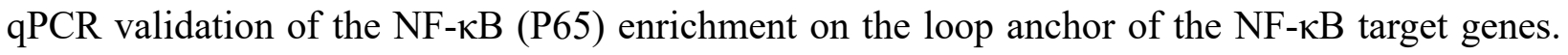

1198 The amount of immunoprecipitated DNA in each sample is represented as signal relative to the 1199 total amount of input chromatin (y-axis). Significant differences were calculated by unpaired one1200 sided t test.

Extended Data Fig. 6 | PD-L1 enhancer identification and knockout. a,b, Histone modification and transcription factor enrichment in the PD-L1 promoter and enhancer region. The transcription factors regulating the PD-L1 gene are marked by arrows. Data are from the UCSC Genome 1205 browser (http://genome.ucsc.edu). c, sgRNAs used in PD-L1 enhancer deletion. Two sgRNAs 1206 were designed for each side of the PD-L1 enhancer. d,e, Validation of homozygous PD-L1 1207 enhancer deletion. f, Genotype of wild-type and PD-L1 enhancer-deleted THP-1 cells as 1208 determined by PCR. 
1210

1211

1212

1213

1214

1215

1216

1217

1218

1219

1220

1221

1222

1223

1224

1225

1226

1227

1228

1229

1230

1231

1232

1233

1234

1235

1236

Extended Data Fig. 7 | Comprehensive map of potential tuberculosis susceptibility genes by integrated eQTL, Hi-C and GWAS multi-omics analysis. a, Circos plot of all reported TB risk SNPs and significant eQTLs associated with risk SNPs, with the corresponding gene expression levels, histone modifications and Hi-C loops on chromosome 12. b, Identification of target genes of TB susceptibility SNPs by integrated eQTL, Hi-C and GWAS multi-omics analysis. c,d, Comparison of the dynamic chromatin loops around the potential targets NSL1 (c) and ASAP1 (d) before and after M.tb infection. The strengthened loops and TB susceptibility SNPs are highlighted by arrow. e, Binding of transcription factors around SNP rs1873613. Data are adapted from the UCSC browser (http://genome.ucsc.edu). f, The mechanism by which the rs1873613 SNP causes TB susceptibility and the role of AdoCbl in the treatment of TB.

Supplementary Table 1. Differentially expressed genes during THP-1 cell differentiation.

Supplementary Table 2. Differentially expressed genes during M.tb infection.

Supplementary Table 3. Key performance metrics of merged sciDLO Hi-C library. In order to distinguish between single cells, we added two rounds of barcodes. In the first round, we added 96 barcodes to the 96-well plate, and in the second round, we added 14 barcodes in the sequencing adapter.

Supplementary Table 4. Key performance metrics of different in situ DLO Hi-C library, including uniquely mapped reads (for mapping efficiency evaluation), non-redundantly mapped reads (for PCR redundancy evaluation), intra- or inter-chromosomal contact (for random ligation noise evaluation), and intra-short- or intra-long-range contact (for the noise of the dangling end and selfligation reads evaluation).

Supplementary Table 5. Dynamics of promoter and enhancer states during THP-1 cell 
1237 differentiation and M.tb infection.

1238

1239 Supplementary Table 6. Loop anchor-located genes associated with chromatin remodeling during 1240 M.tb infection.

1241

1242 Supplementary Table 7. Integrated GWAS summary and eQTL information.

1243

1244 Supplementary Table 8. Barcoded linker, MGI-2000 platform sequence adapter, and barcoded 1245 amplification primer used in sciDLO Hi-C library construction.

1246

1247

1248

1249 

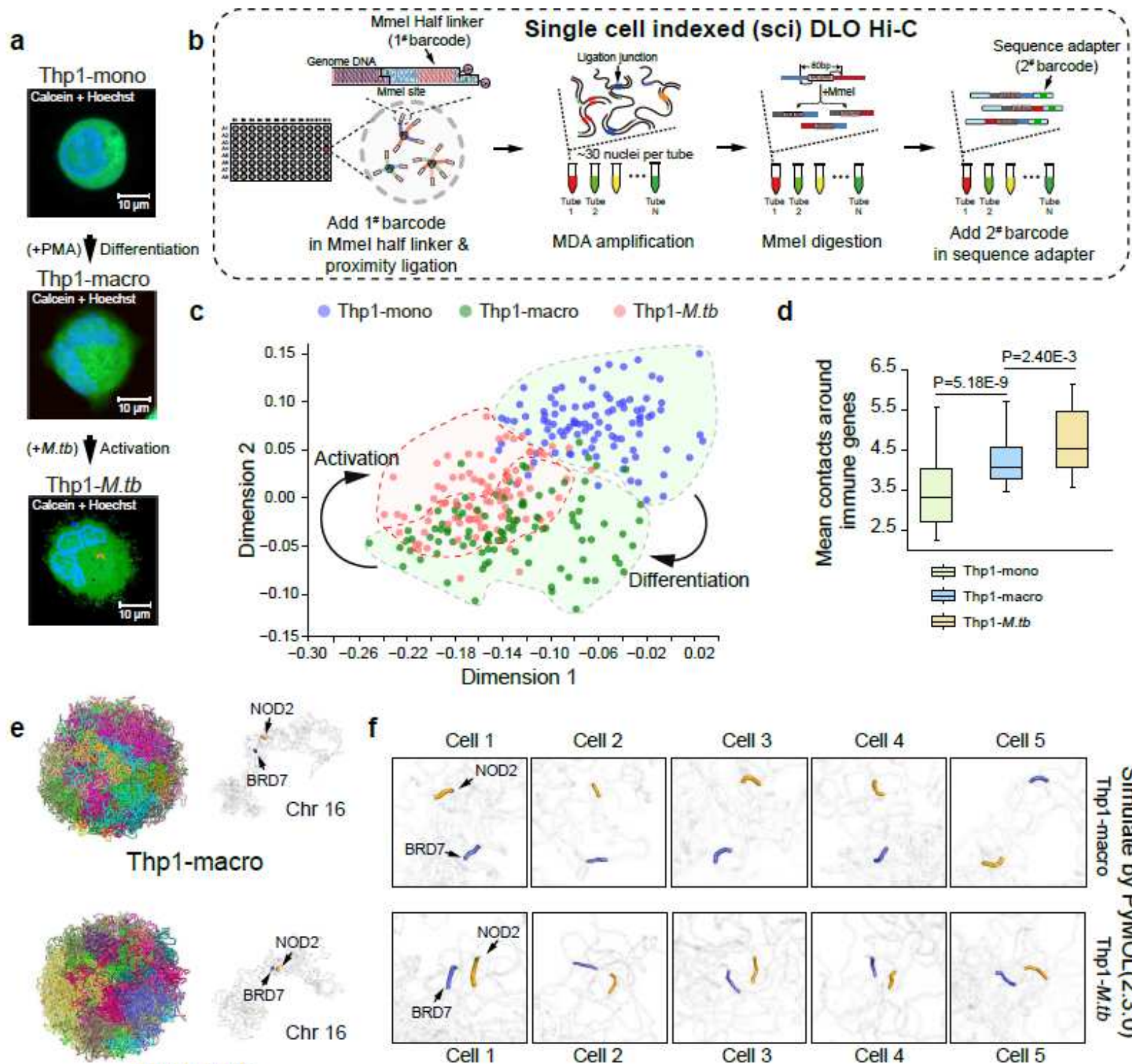

Thp1-M.tb

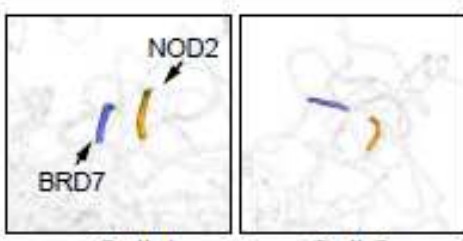

Cell 1
Cell 2

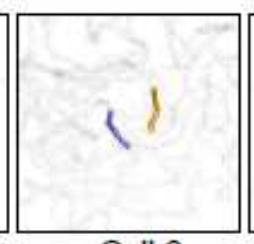

Cell 3

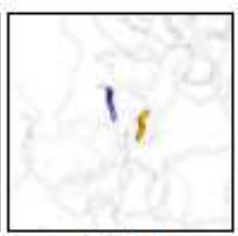

Cell 4
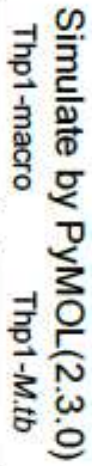

Cell 5

\section{Figure 1}

Decoding the 3D Genome of monocytes, macrophages, and M.tb infected macrophages with single cell indexed (sci) DLO Hi-C. a, Representative calcein and hoechst fluorescence staining images of Thp1mono, Thp1-macro, and Thp1-M.tb cells. b, Flowchart of the sciDLO Hi-C method. c, Present cluster analysis result of sciDLO Hi-C data sets by using two-dimensional scatter plots. d, Comparison of average contacts around immune genes $( \pm 10 \mathrm{~Kb}$ around TSS sites) between Thp1-mono, Thp1-macro, and Thp1M.tb cells. e, Simulation of chromatin three-dimensional conformation of representative immune highly 
activated and moderately activated cells. f, Comparison of the spatial location of NOD2 and BRD7 in immune highly activated and moderately activated cells by using simulated nucleus. NOD2 and BRD were marked with arrows.
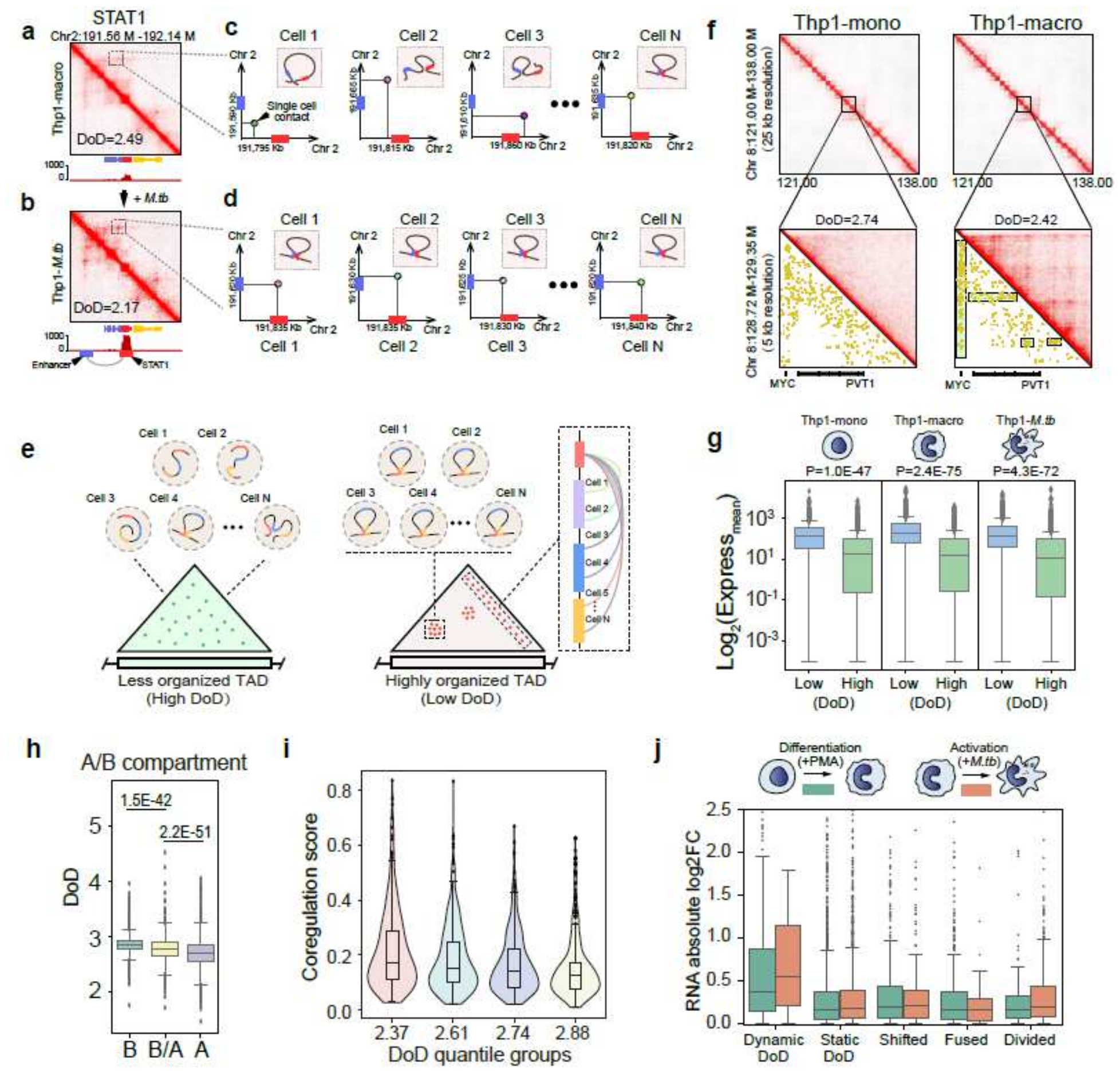

j
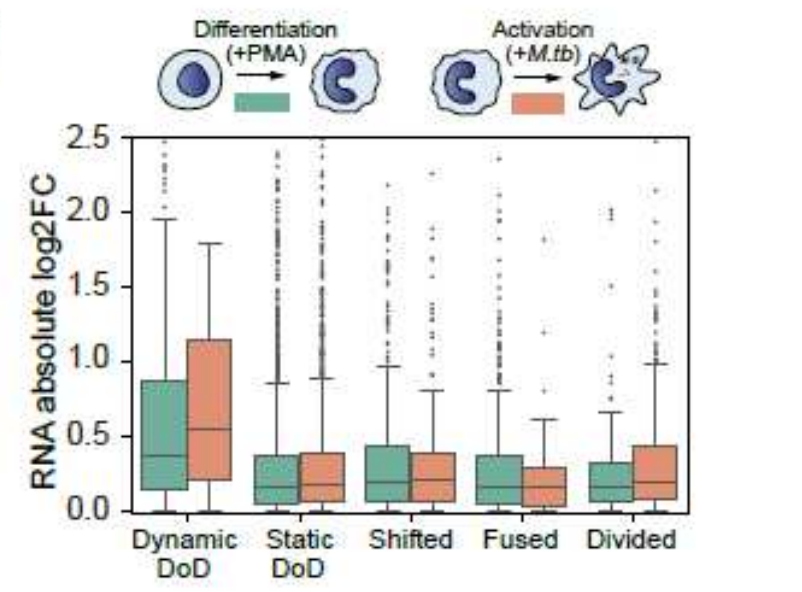

\section{Figure 2}

Evaluating the order and stochasticity of single cell chromatin folding with degree of disorder (DoD). $a, b$, Bulk cell chromatin contact matrix and gene expression level of STAT1 TAD of Thp1-macro and Thp1M.tb cells. The chromatin interaction hot spot which formed in low DOD TAD were marked by dashed box. Chromatin loop mediated STAT1 and enhancer interaction were labeled by arrows. c,d, Single cell 
chromatin contacts around STAT1 gene of Thp1-macro and Thp1-M.tb cells. Individual cells have similar chromatin folding patterns in the low DoD TAD. e, Illustration of the TAD degree of disorder (DoD) which was used to measure the order and consistency of chromatin folding in single cells. $f$, DoD values of Thp1-mono and Thp1-macro around MYC gene region by using bulk cell DLO Hi-C data sets. $\mathrm{g}$, Relationship between TAD DoD and average gene expression level (normalized read count) in Thp1mono, Thp1-macro, and Thp1-M.tb, respectively. The DoD value higher than median was defined as "High DoD"; lower than the median was defined as "Low DoD". Significance of differences was measured by Kolmogorov-Smirnov test. $h$, Correlation between the TAD DoD and A, B compartments. In $x$ axis, B/A means the TADs which contains both $A$ and B compartments. Significance of differences was measured by unpaired one-sided t test. i, Correlation between the TAD DoD and coregulation score (CRS). TADs with lower TAD DoD preferentially have higher CRS, underlying more coordinated gene regulation in such TADs. $x$ axis is the average value of the quantile groups with the lowest, low, high and highest TAD DoDs, respectively. j, Absolute values of the log2 fold changes in gene expression (y-axis) in the boundary not changed but DoD value significant changed TADs (dynamic DoD), boundary and DoD unchanged TADs (static DoD), boundary shifted TADs (shifted), boundary fused TADs (fused), and boundary divided TADs (divided) during differentiation and infection. 


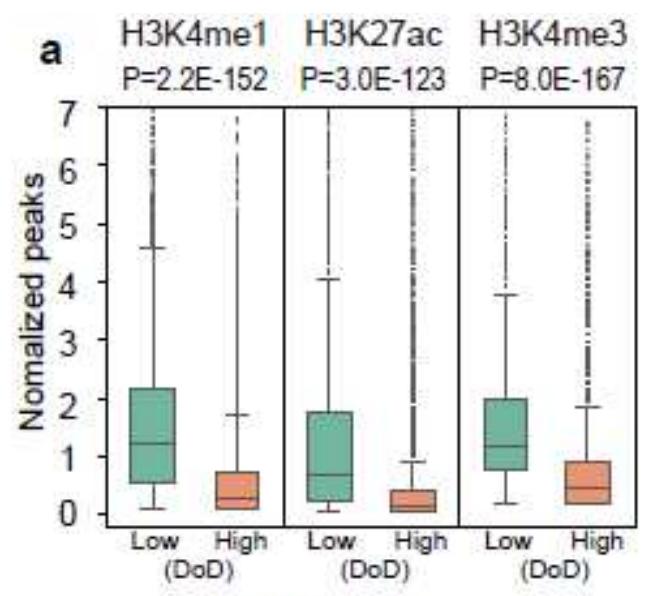

Thp1-macro

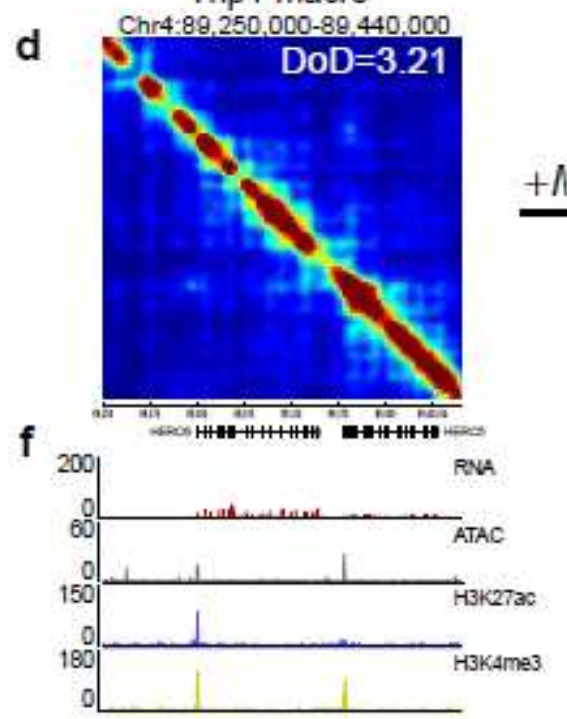

h

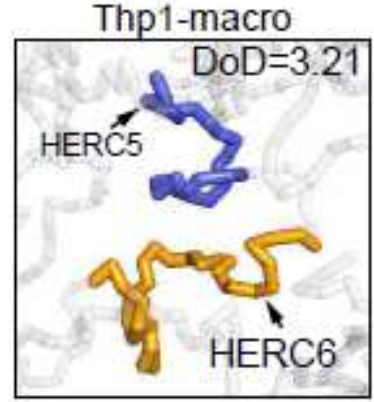

b

$$
\text { H3K27me3 H3K9me3 }
$$

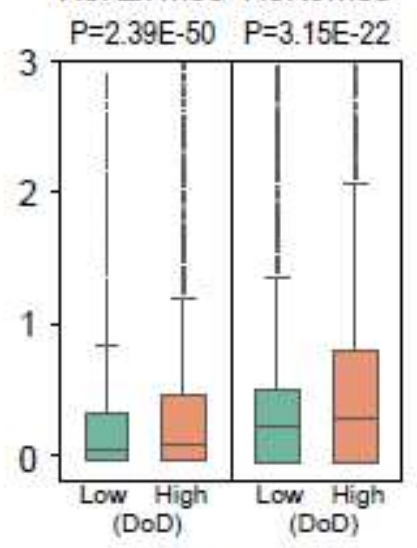

Thp1-M.tb

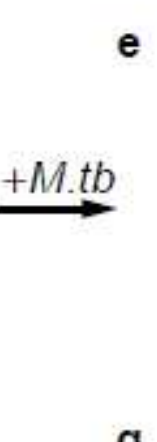

g
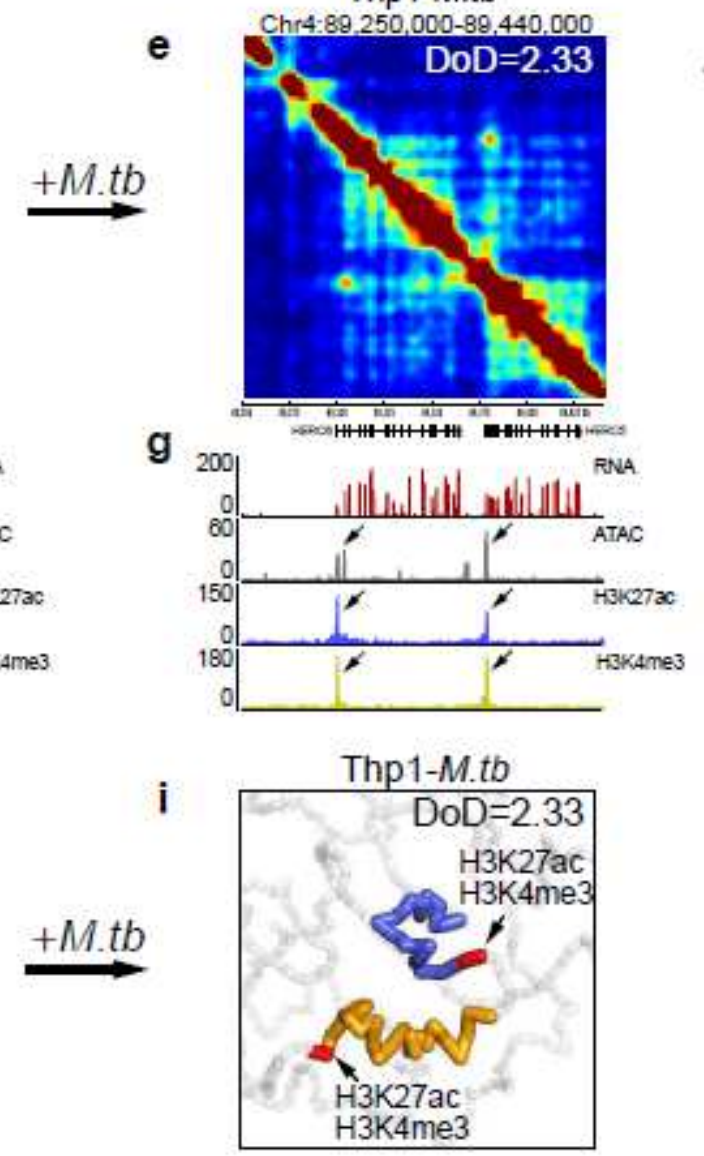

c

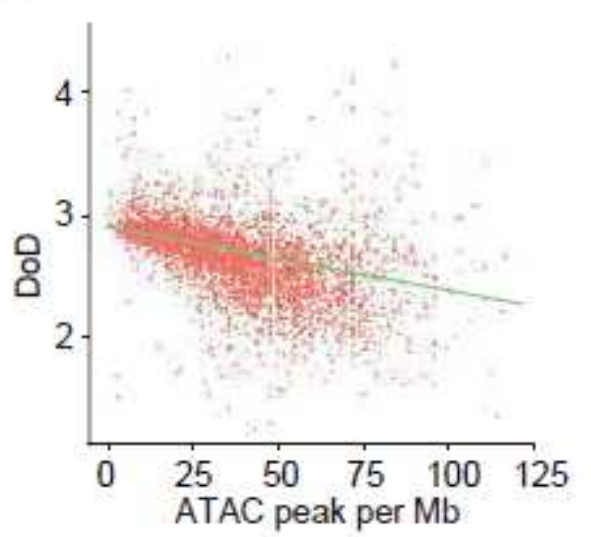

ATAC peak per $\mathrm{Mb}$

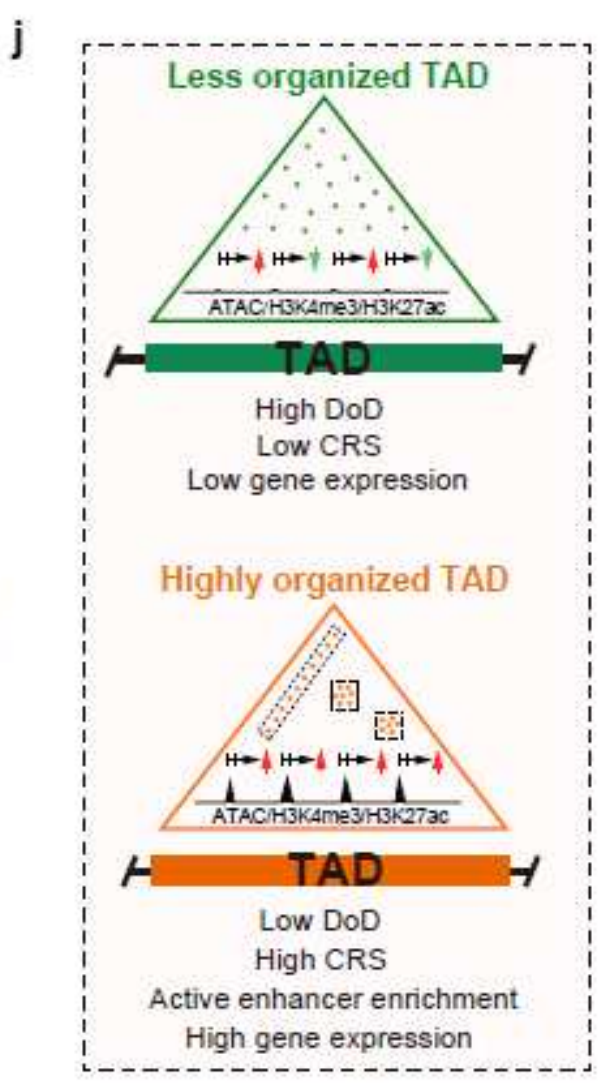

\section{Figure 3}

Low DoD is associated with active epigenetic modification and high gene expression level. a,b, Correlation between TAD DoD and chromatin epigenetic profile. The y axis shows the normalized ChIPseq peaks per TAD. The DoD value higher than median was defined as "High DoD"; lower than the median was defined as "Low DoD”. Significant differences were measured by unpaired one-sided t test. c, Correlation between TAD DoD and chromatin accessibility. Each dot represents one TAD. $x$-axis is the number of ATAC peaks per Mb. d,e, Chromatin contact matrix and DoD value changes around HERC5 and HERC6 gene region after M.tb infection. f,g, RNA expression level, chromatin accessibility, H3K27ac, and H3K4me4 histone modification changes after M.tb infection. h,i, Simulated three-dimensional structure of 
HERC5 and HERC6 gene region during M.tb infection by using bulk cell DLO Hi-C data sets. j, The relationship between DoD value, chromatin folding order, chromatin accessibility, gene transcription coregulation, and histone modification.

a
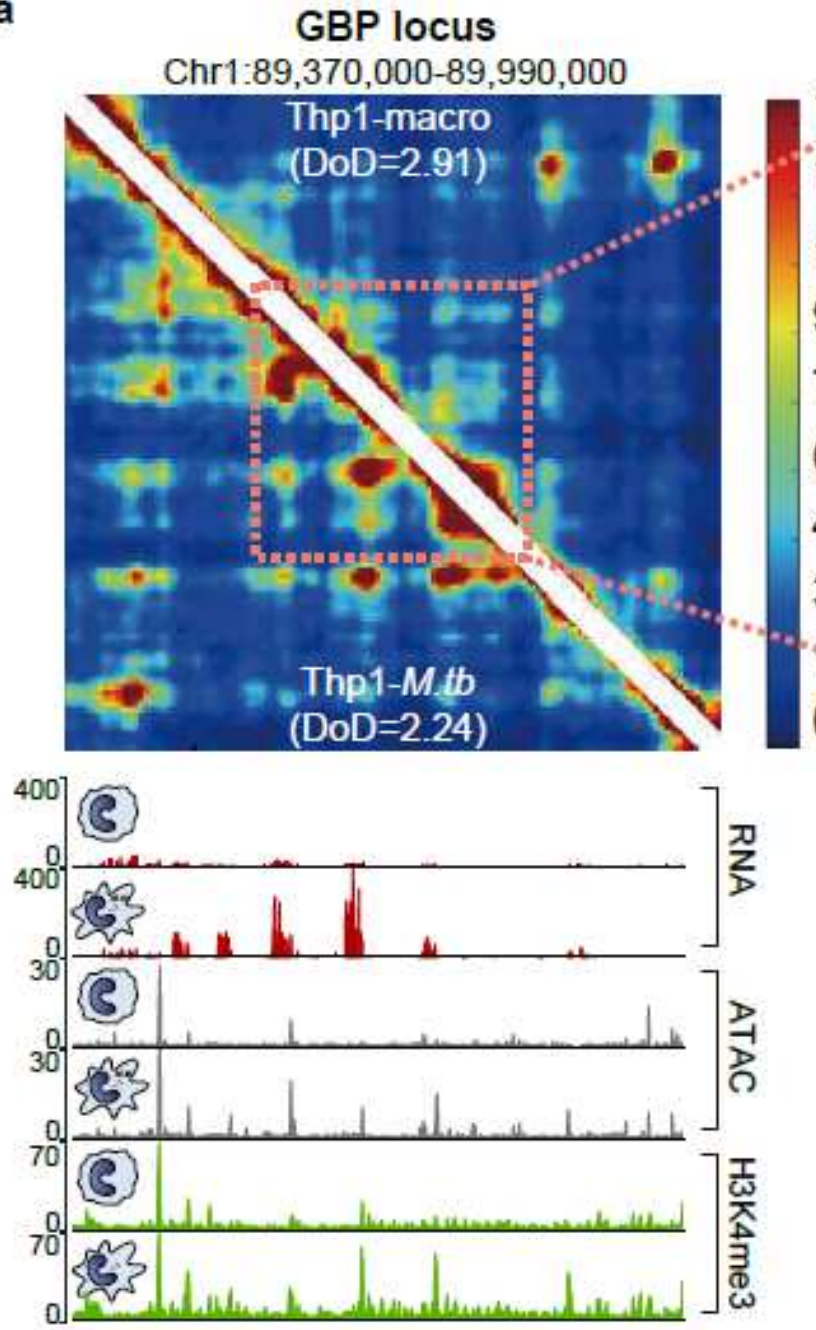

d
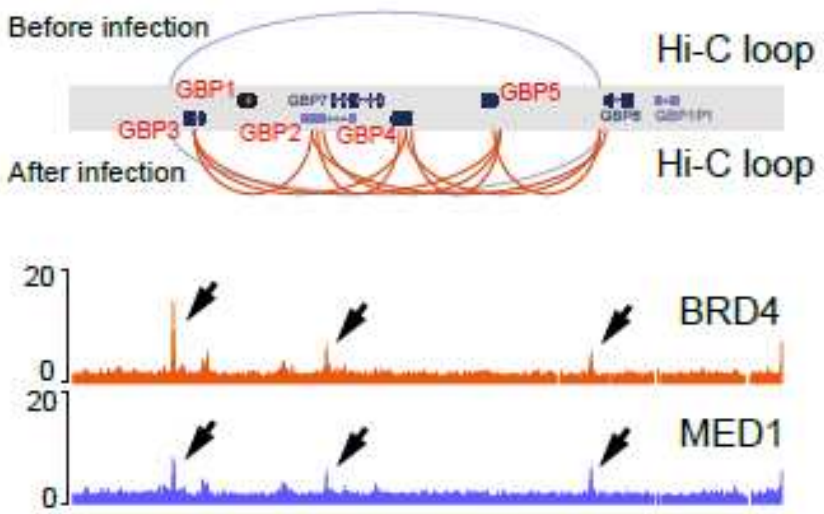

\section{Merged single cell contact matrix}

b Chr1:89575000-89800000
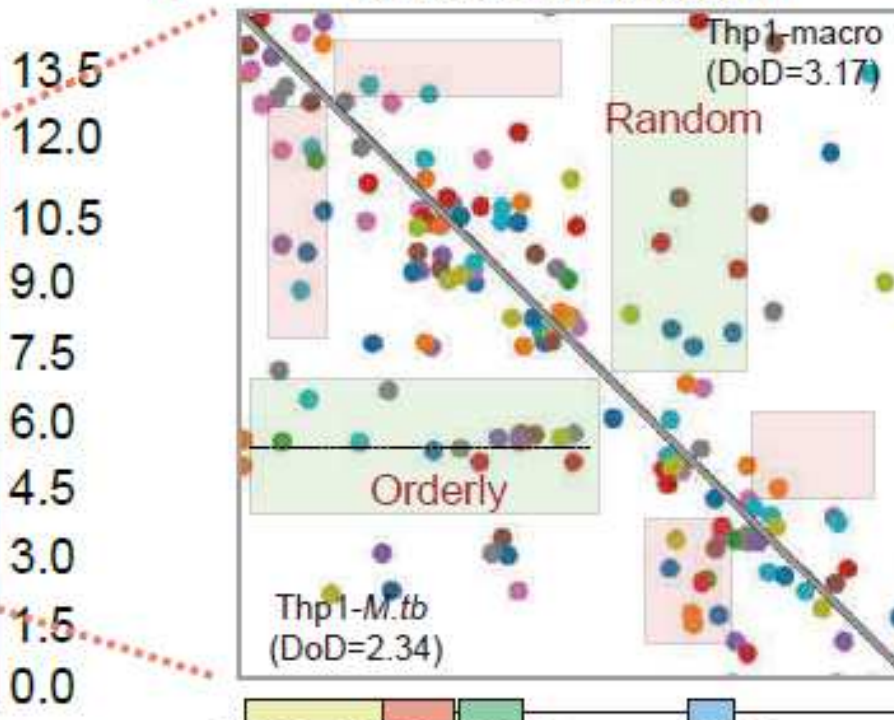

\section{Random}

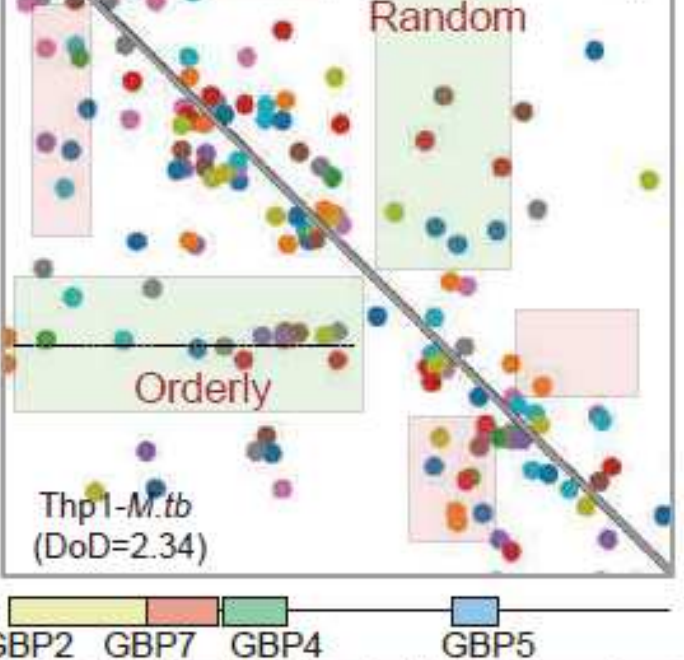

c
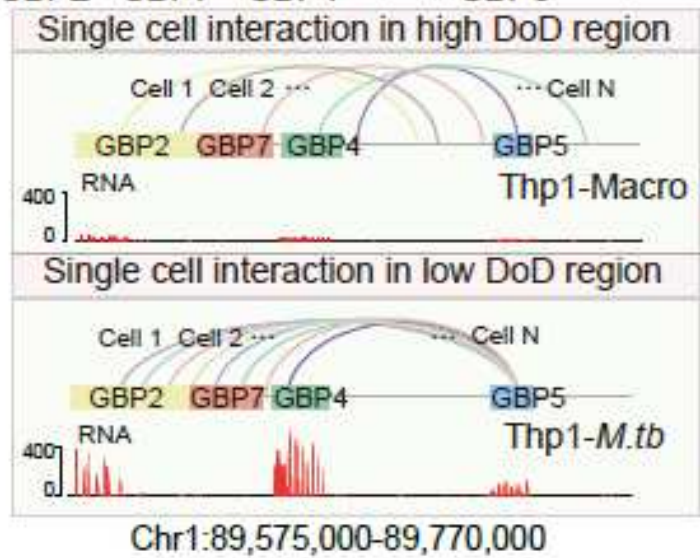

e

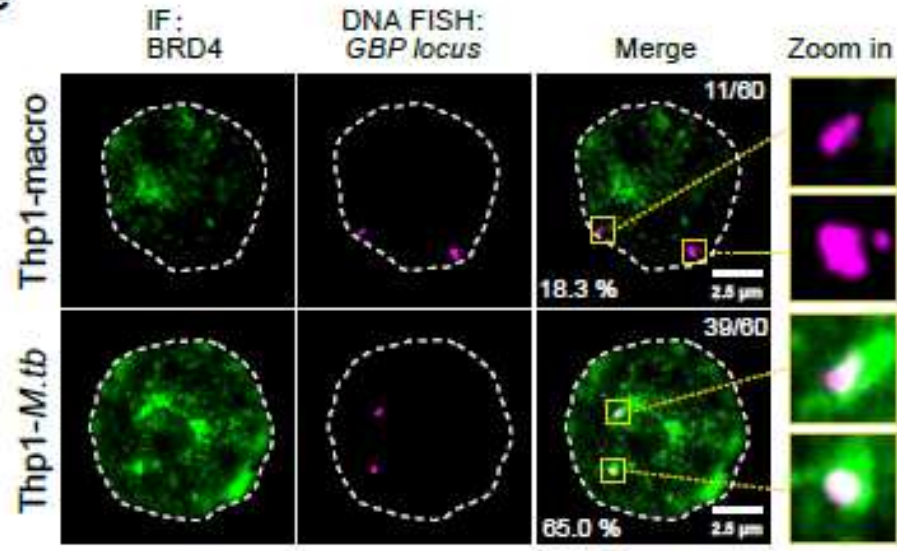

Figure 4

Remodeling of the chromatin configuration of guanylate-binding protein (GBP) gene clusters. a, Comparison of TAD DoD value, chromatin interaction matrices, chromatin loops, RNA expression levels 
and epigenetic modifications around the GBP loci in bulk cells before and after M.tb infection. b, Comparing the single cell chromatin contact differences using merged single cell chromatin contact matrix. Each color of the dots in the matrix represents the chromatin contact from the same cell. c, Single cell chromatin contacts between GBP2, GBP4, GBP5, and GBP7 and RNA expression before and after M.tb infection. d, Depiction Hi-C chromatin loops before and after infection and BRD4 and MED1 ChIPseq peaks around GBP gene clusters. e, Co-localization between BRD4 and the GBP gene cluster by IF and DNA-FISH in Thp1-macro and Thp1-M.tb cells. IF, DNA-FISH, and merged channels (overlapping signal in white) are shown in separate images. The dashed line highlights the nuclear periphery, determined by DAPI staining. The rightmost column shows the area in the yellow box in greater detail. Each cell type, we counted 60 GBP gene loci, 18.3\% (11/60) GBP loci were co-located with BRD4 in Thp1-macro cells and $65.0 \%(39 / 60)$ GBP loci were co-located with BRD4 in Thp1-M.tb cells.

\section{Fig. 5}

a Thp1-mono

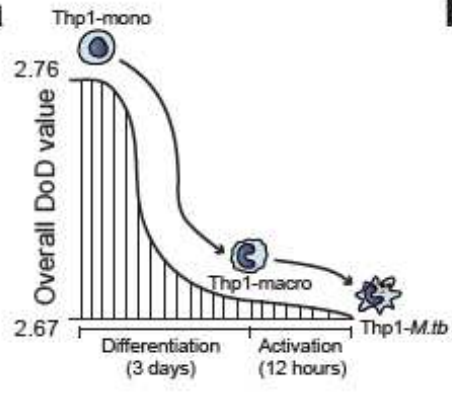

e

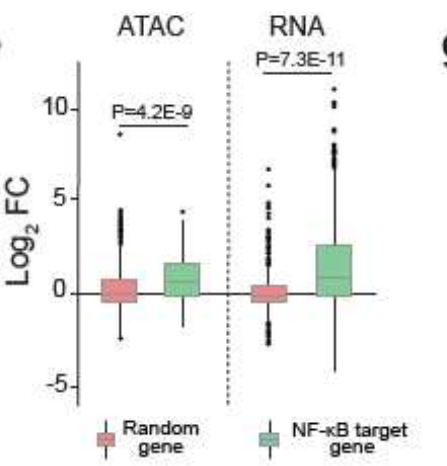

$\mathbf{f}$

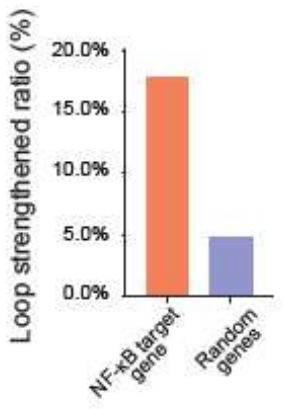

b

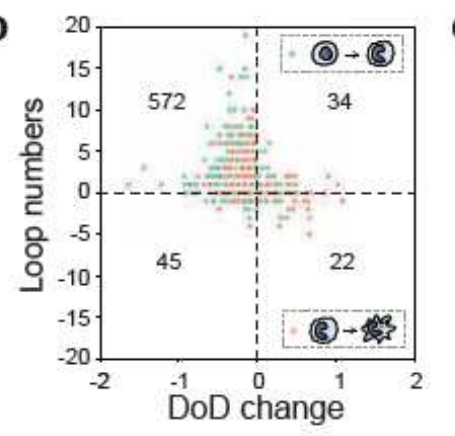

- Strengthened loop anchor gene
- Random gene

C

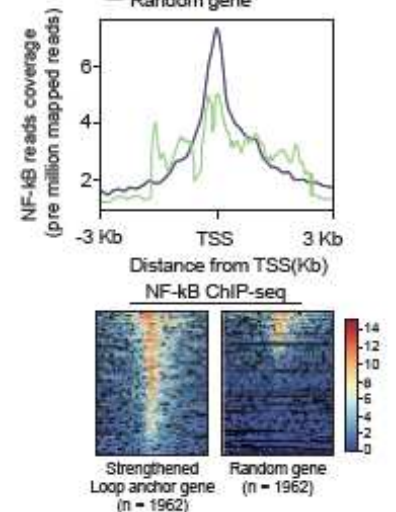

d

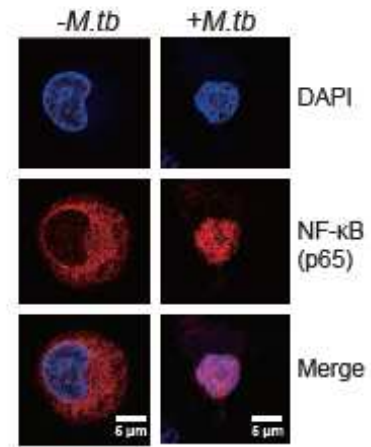

g

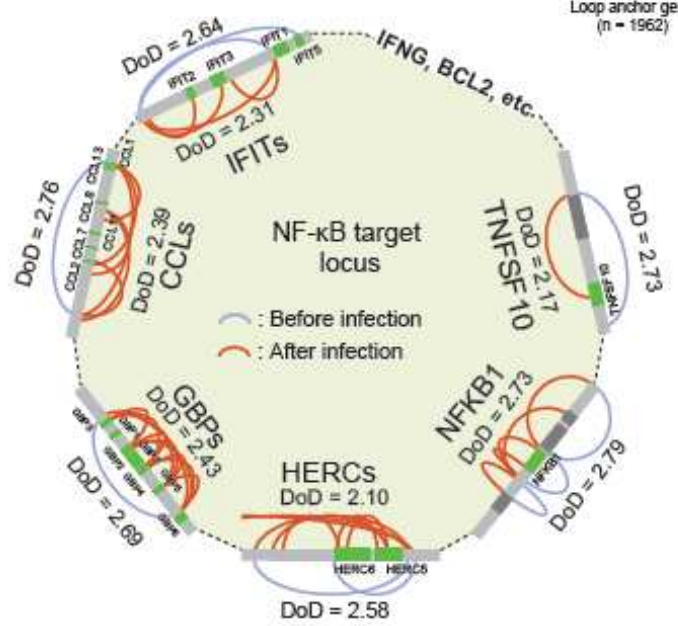

h
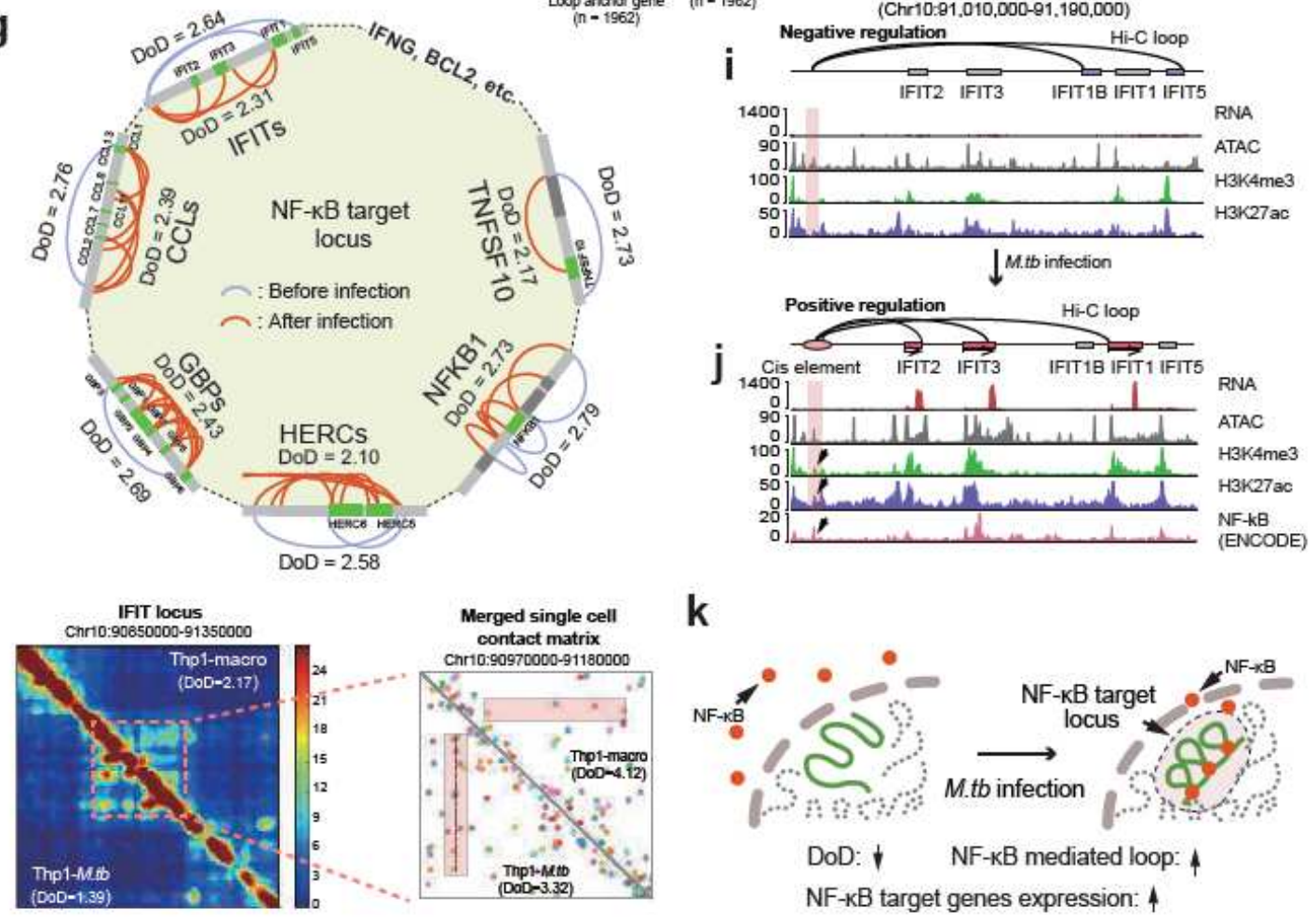

k

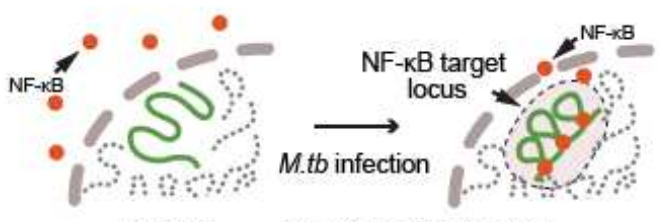

DoD: NF-kB mediated loop: $\uparrow$

$\mathrm{NF}-\mathrm{kB}$ target genes expression: $\uparrow$

Figure 5 
Reorganization of chromatin architecture around NF-KB target gene sites. a, Overall TAD DoD of THP-1 cells during differentiation and infection. The $y$-axis represents the average DoD value of TADs in each state. $b$, The relationship between TAD DoD and chromatin loop during differentiation and activation. The $x$-axis represents log2 fold change of TAD DoD value, and the $y$-axis represent the number of loop changes in each TAD. c, Compare NF-KB enrichment around transcription start site $( \pm 3 \mathrm{~Kb})$ between strengthened loop anchor gene and random control gene. The gene in the heatmap were sorted according to the enrichment intensity of NF-KB. ChIP-seq data is from ENCODE (GM12891 cell line). d, Immunofluorescence analysis of NF-KB (p65) subcellular location before and after M.tb infection. e, Log2 fold changes of ATAC peaks and RNA expression level of NF-KB target genes $(n=435)$ and random control genes. Significant differences were calculated by unpaired one-sided $t$ test. $f$, The ratio of strengthened loop/total loop of NF-KB target gene sties and random control sites. g, Chromatin loop remodeling and TAD DoD changes in typical NF-KB target gene loci. h, Bulk cell and merged single cell chromatin interaction matrices around IFIT gene locus. Cells with more consistent chromatin interaction were marked with a dashed line. i,j, Comparison of chromatin loop configurations, RNA expression levels, and epigenetic modifications of the IFIT gene family locus before (i) and after (j) M.tb infection. The arrows in (j) indicate the potential cis element linked with IFIT2, IFIT3, and IFIT1. k, Schematic diagram of chromatin remodeling of NF-KB target genes during infection.

a

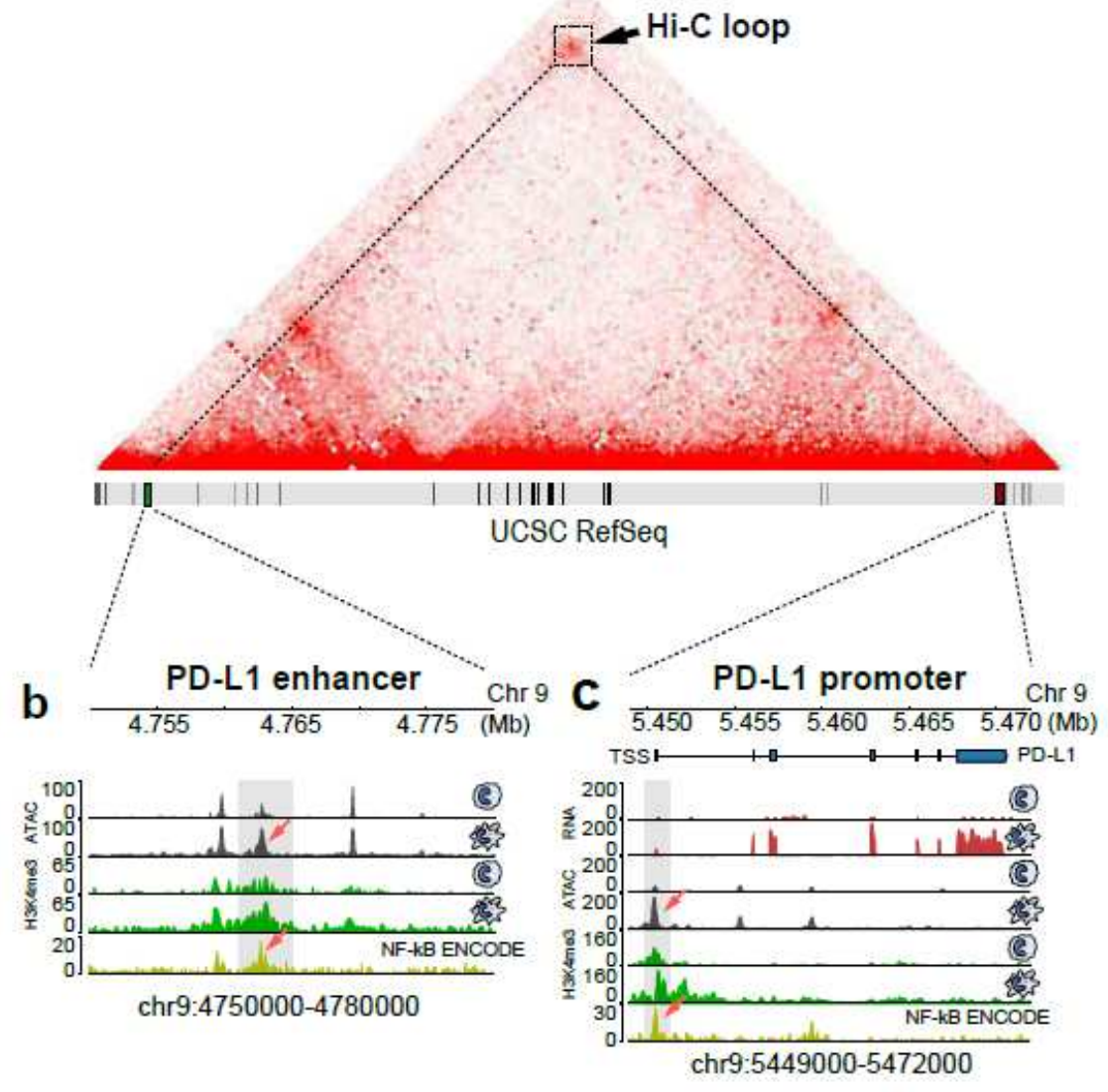

d
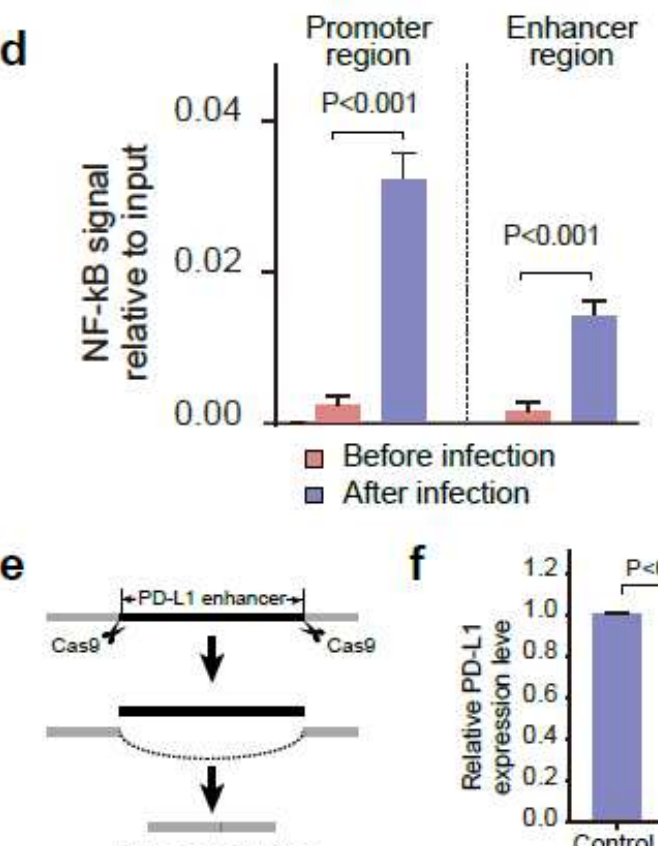

Enhancer deletion

g

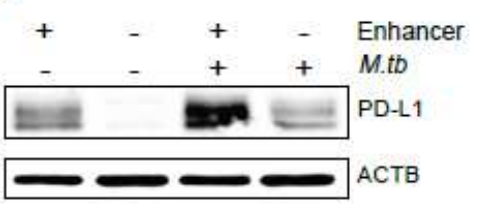

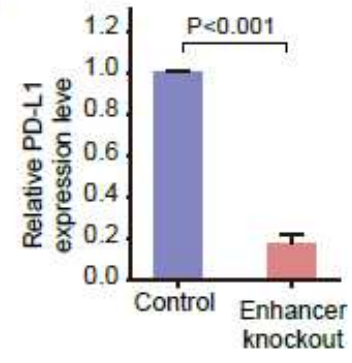

h

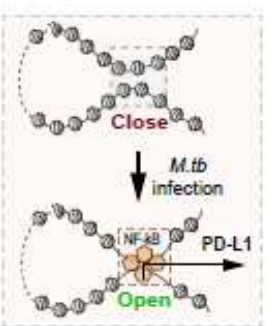

Figure 6 
Functional identification of PD-L1 enhancer. a, Interaction of the PD-L1 enhancer and promoter in the Hi-C matrix. b,c, Genome Browser view of RNA expression levels, chromatin accessibility, and histone modifications at the PD-L1 gene and enhancer regions in Thp1-macro and Thp1-M.tb cells. NF-KB ChIPseq peaks (ENCODE, GM15510 cell line) and strengthened ATAC-seq peaks were marked by arrows. d, ChIP-qPCR validation of the NF-KB (P65) enrichment on the PD-L1 enhancer and promoter regions. The amount of immunoprecipitated DNA in each sample is represented as signal relative to the total amount of input chromatin (y-axis). Significant differences were calculated by unpaired one-sided t test. e, Experimental design of sgRNA-guided enhancer perturbation by the Cas 9 protein. $f$, Relative mRNA expression levels of PD-L1 in Thp1-macro cells and the same line after enhancer deletion. Error bars show mean $\pm S E$ of results of three replicates; the difference was assessed using unpaired one-sided $t$ test. $g$, PD-L1 protein levels in Thp1-macro cells and the same cell line after enhancer deletion before and after M.tb infection. h, Schematic of enhancer-promoter interaction mediated regulation of PD-L1 expression during M.tb infection.

a

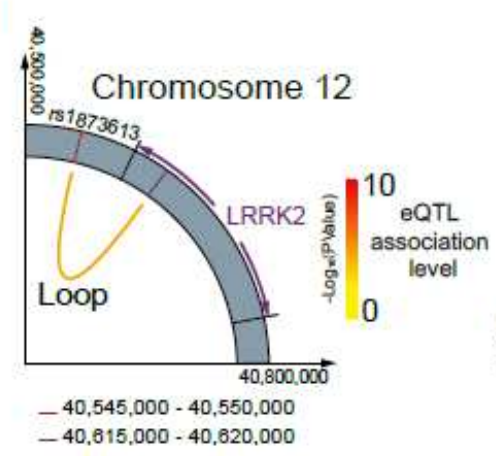

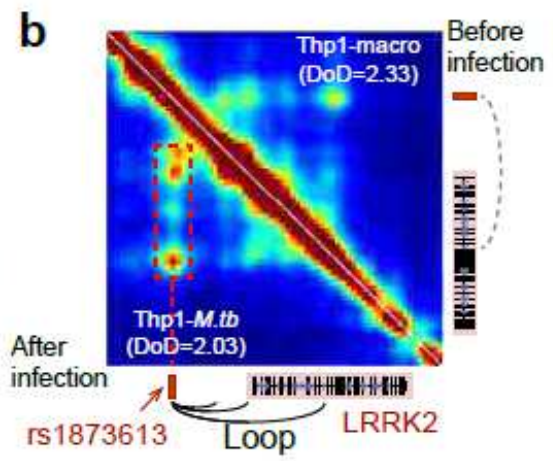

e
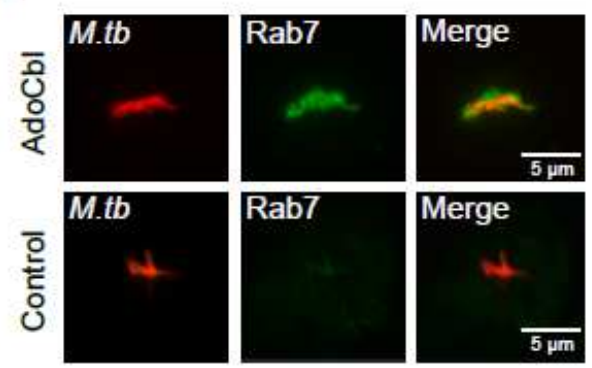

h

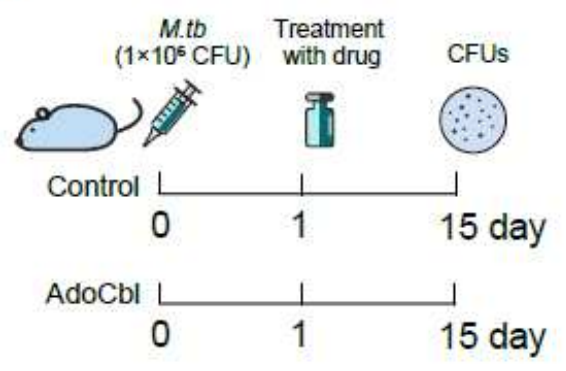

f
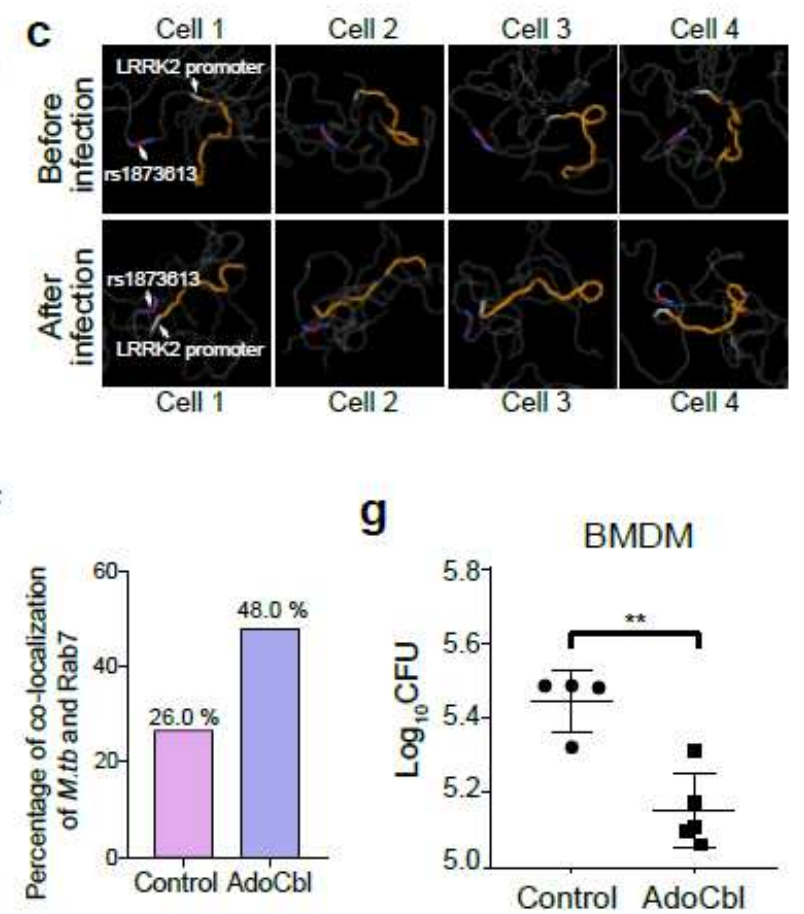

g
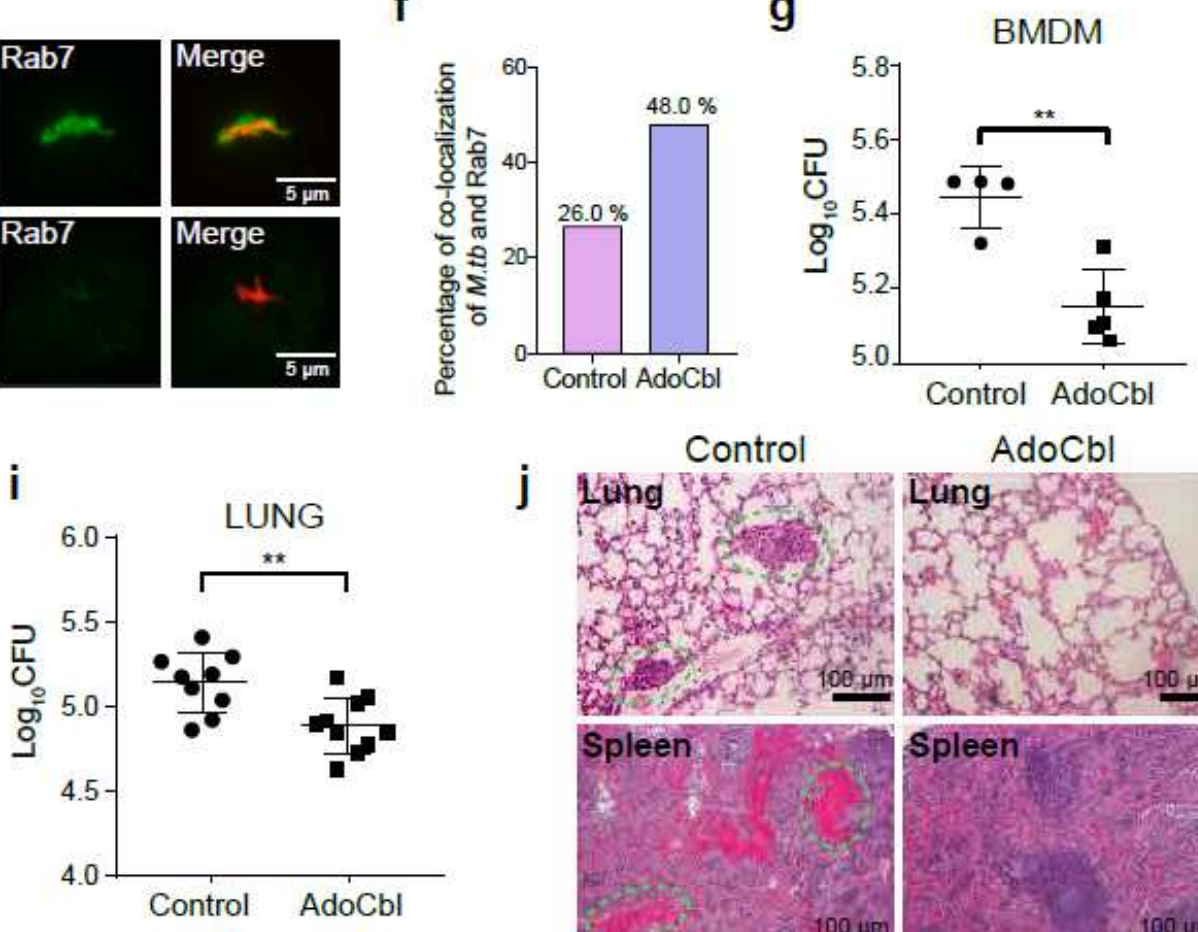

Control

AdoCbl

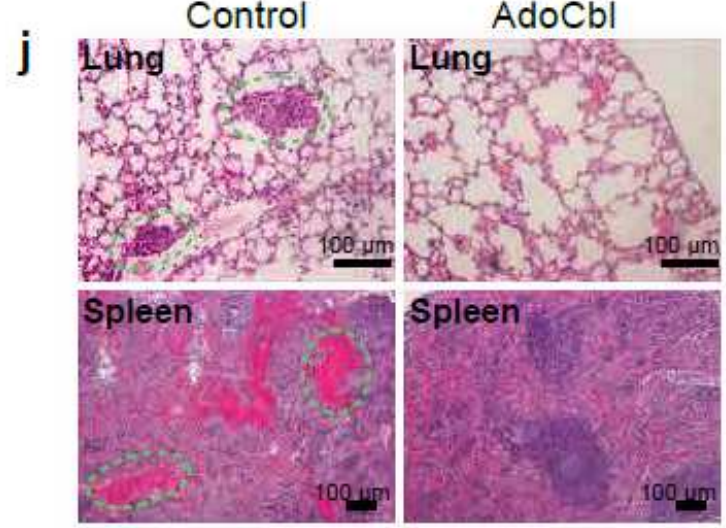

Figure 7 
Identification of long range regulatory target genes of TB susceptibility loci and novel anti-TB drug targets by integrated multi-omics analysis. a, Identification of long range regulatory target genes of TB susceptibility loci, LRRK2 by integrated eQTL, Hi-C and GWAS multi-omics analysis. b, The rs 1873613 SNP is located in the anchor of the LRRK2 gene chromatin loop, which is significantly strengthened after M.tb infection, as shown in the Hi-C matrix. c, Simulated typical single cell chromatin structure of SNP rs1873613 and LRRK2 gene region before and after M.tb infection. $d$, Regulation of LRRK2 gene expression by the rs 1873613 SNP. The $x$ axis indicates three genotypes of individuals from the GenotypeTissue Expression (GTEx) dataset. e,f, Immunofluorescence analysis and quantification of the colocalization of the M.tb (H37Ra-RFP) and Rab7 in bone marrow-derived macrophages treated with the LRRK2 inhibitor AdoCbl and PBS (control). g, CFU assays of M.tb in bone-marrow-derived macrophage cells treated with AdoCbl and PBS (control). Significant differences were calculated by unpaired onesided $t$ test. h,i, CFU assays of M.tb in C57BL/ 6 mouse lungs treated with AdoCbl and PBS (control). Significant differences were calculated by unpaired one-sided $t$ test. j, HE-stained lung and spleen sections in the AdoCbl and control (PBS) groups. Obvious lesions in the lungs and spleen are highlighted by the dashed lines.

\section{Supplementary Files}

This is a list of supplementary files associated with this preprint. Click to download.

- NSMCFigS1S7.pdf

- Tables1ThpThpp.xlsx

- TableS2THPPRa.xlsx

- TableS3sciDLOHiCdata.pdf

- TableS4BulkcellHiCdata.pdf

- Tables5promoterstatechange.xlsx

- TableS6Loopanchorgene.xlsx

- TableS7SNPinformation.xIsx

- TableS8Linkeradapterandprimer.xlsx 